\title{
Newton's Second Law of a particle with variable mass
}

\author{
M.T. Thomaz ${ }^{* 10}$ \\ ${ }^{1}$ Universidade Federal Fluminense, Niterói, RJ, Brasil.
}

Received on March 09, 2021. Accepted on March 22, 2021.

\begin{abstract}
Abordamos a questão do movimento de uma partícula com massa variável observada a partir de um referencial inercial. Consideramos duas situações diferentes de corpos com massa variável: $(i)$ o valor de massa intrínseca da partícula varia ao longo do tempo; (ii) consideramos um sistema de partículas cujo o valor da massa do centro de massa $(\mathrm{CM})$ varia, sendo que o conjunto é formado por partículas, essas com massa constante, mas cujo o número de partículas que o constituem varia no tempo. Mostramos que a Segunda Lei de Newton distingue o caso em que a massa intrínseca da partícula varia ao longo tempo da situação em que temos sistemas compostos de partículas cuja massa total varia com o tempo. No primeiro caso, estudamos as consequências da equação de movimento de um partícula com massa variável não ser covariante em referenciais inerciais sob as transformações galileanas. Nós também mostramos que a equação que descreve a dinâmica do CM de um sistema com número variável de partículas preserva a equivalência de todos os referenciais inerciais sob as transformações galileanas. Verificamos a não conservação do vetor de momento linear do CM de um conjunto de partículas livres durante o tempo em que uma partícula sai ou entra no sistema.

Palavras-chave: Mecânica, Segunda Lei de Newton, partícula com massa variável, partícula com massa intrínsica variável, referencial inercial, centro de massa (CM) de um sistema com massa variável, momento linear, energia mecânica.
\end{abstract}

We approach the question of the movement of a particle with variable mass observed from an inertial frame. We consider two different situations: $(i)$ a particle whose intrinsic mass value varies over time; $(i i)$ the center of mass $(\mathrm{CM})$ of a set of particles with constant mass but with a variable number of particles belonging to it. We show that Newton's Second Law distinguishes the case in which the intrinsic mass of the particle varies over time from systems composed of particles, with constant mass, whose total mass varies over time. In the first case, we study the consequences of the equation of motion of a particle with variable mass is not covariant in inertial references under Galilean transformations. We also show that the equation that drives the dynamics of the $\mathrm{CM}$ of a system with variable number of particles preserves the equivalence of all inertial frames under the Galilean transformations. We verify the non-conservation of the linear momentum vector of the CM of a set of free particles during the time that one particle leaves or comes into the system.

Keywords: Mechanics, Newton's Second Law, particle with variable mass, variable intrinsic mass of the particle, inertial frame, center of mass (CM) of systems with variable mass, linear momentum, mechanical energy.

\section{Introduction}

The three laws stated by Sir Isaac Newton in his "Philosophiae Naturalis Principia Mathematica" in 1687 [1] describe the movement of the classical particles and extended bodies. Their dynamics are driven by Newton's Second Law. The mass of the known particles in Nature are constant, although the mass of extended bodies may vary over time.

When we think of a body that has a variable mass, systems like this appear in our minds: rockets that release fuels, mineral deposit conveyor, a chain reaching the ground and other similar bodies.

In A. Sommerfeld's original proposal [2] to obtain the dynamic equation of a body with variable mass during its movement he uses the conservation of the total linear momentum of the entire system frame of reference of this

\footnotetext{
* Correspondence email address: mariateresa.thomaz@gmail.com
}

body. Since then, in the 60 's we have some articles that present a framework to describe systems with variable masses [3, 4. More recently, this issue has been revisited by some authors [5]. We can find a more complete list of references on the dynamics of bodies with variable mass in those last articles. All the articles look for the equation that describes correctly the dynamics of a system whose mass varies with time. In these systems, their masses vary over time, although they are make up of particles with constant masses. In the previous references, the authors apply the Impulse-Momentum Theorem or the conservation of the linear momentum of the system in the body reference frame whose mass varies during its movement.

In these mentioned references, the equations that describe the dynamics of extended bodies with variable masses are not obtained directly from Newton's Second Law applied to particles of variable mass. It is stated that, in the case of particles with variable mass that the 
equation of motion obtained from Newton's Second Law is not invariant under the transformations of Galileo [8]. However, this general statement in Newton's Second Law applied to particles of variable mass does not explain how would be the movement of a particle with intrinsic variable mass if we assume that Newton's Second Law is valid for this type of particle.

In 2019, Thomaz and Corrêa Silva 9] studied the dynamics of the center of the mass (CM) of a system with variable mass. They applied Newton's Second Law to each particle, in a inertial frame, to derive the CM movement from this system. They considered two physical situations where we have the mass of the particle set varies over time: $(i)$ each particle in the set has constant mass but the number of particles that make up the system varies in time; $(i i)$ the mass of one of the particles, that belong to the set, varies over time.

An interesting question to be addressed here is whether Newton's Second Law distinguishes the aforementioned composite system, with variable mass, from particles whose intrinsic mass varies over time. This issue has not been discussed in undergraduate Physics textbooks. It is always interesting to challenge undergraduate students with new situations in addition to traditional books, where they have to apply the basic laws of physics.

In the present article, we have two aims: $(i)$ to study the consequences of Newton's Second Law in describing the motion of a particle whose intrinsic mass varies over time; (ii) to verify if the equation derived in reference 9 for the movement of a CM of a set of particles of constant mass and whose number of components varies over time of one unit is covariant in all inertial frames under the Galilean transformations. The discussion of these two items will allow us to answer the question previously presented.

As we said before, we still do not know any particle in the Nature whose mass varies over time. However, it is interesting to explore the consequences of the laws that we believe that could describe the Nature.

In section 2 we study the dynamics of a particle with variable intrinsic mass. We study the validity of Newton's three laws in describing the motion of this type of particles when they are observed from different inertial frames. In subsections 2.1 and 2.2 we bring our attention to the linear momentum vector and to the mechanical energy of particles whose intrinsic mass varies with time. In section 3 we follow the dynamics of the CM of a system of particles, with constant masses, but with a variable number of elements that belong to it. We consider the simplest set with $N=2$ particles. We show in this case that the CM equation of motion of the particle set is invariant under Galileo's transformations. We also discuss the behaviour of the linear momentum vector of the CM when its particles are free. In section 4 we have our conclusions. In Appendix A we present the solutions of Newton's Second Law in a particular inertial frame for some special forces.

\section{Newton's three laws applied to a particle with variable mass}

The measured values of the kinematic quantities of a given particle depends of the reference frame where the observer is at rest. Two or more observers who have relative movement among themselves can follow and measure the position, velocity and/or acceleration of the same particle. For velocities $v$ much smaller than the velocity of light $c$, that is, $v \ll c$, with $c=299.792 \frac{\mathrm{km}}{\mathrm{s}}$, the relationship between the values of the kinematic quantities of a given particle, measured in distinct frames, is given by the Galilean transformations [10:175].

In Fig. 1 we draw the coordinate axes that are fixed in the frames $\mathbf{S}$ and $\mathbf{S}^{\prime}$. The frame $\mathbf{S}^{\prime}$ moves with velocity $\overrightarrow{\mathbf{V}}(t)$ relative to frame $\mathbf{S}$. The coordinate axes $x y z\left(x^{\prime} y^{\prime} z^{\prime}\right)$ are fixed in the frame $\mathbf{S}\left(\mathbf{S}^{\prime}\right)$. $\mathbf{O}\left(\mathbf{O}^{\prime}\right)$ is the origin of the set of coordinates $x y z\left(x^{\prime} y^{\prime} z^{\prime}\right)$. Vector $\overrightarrow{\mathbf{R}}(t)$ gives the position of the origin $\mathbf{O}^{\prime}$ with respect to the origin $\mathbf{O}$.

Let $\overrightarrow{\mathbf{r}}(t)$ be the position vector of the particle $\mathbf{P}$ with respect to the origin $\mathbf{O}$, fixed in reference frame $\mathbf{S}$, at instant $t$. The vector position of the same particle measured from the origin $\mathbf{O}^{\prime}$ of the frame $\mathbf{S}^{\prime}$, in the same instant is $\overrightarrow{\mathbf{r}}^{\prime}(t)$.

Figure 1 permits to write down the Galilean transformations of the position vectors of the same particle measured from the distinct frames $\mathbf{S}$ and $\mathbf{S}^{\prime}$,

$$
\overrightarrow{\mathbf{r}}(t)=\overrightarrow{\mathbf{r}}^{\prime}(t)+\overrightarrow{\mathbf{R}}(t) .
$$

From eq. (1) we obtain the relationship between the velocity vectors of the particle measured in frame $\mathbf{S}$, $\overrightarrow{\mathbf{v}}(t)=\frac{d \overrightarrow{\mathbf{r}}(t)}{d t}$, and its velocity vector measured in frame $\mathbf{S}^{\prime}, \overrightarrow{\mathbf{v}}^{\prime}(t)=\frac{d \overrightarrow{\mathbf{r}}^{\prime}(t)}{d t}$

$$
\overrightarrow{\mathbf{v}}(t)=\overrightarrow{\mathbf{v}}^{\prime}(t)+\overrightarrow{\mathbf{V}}(t) .
$$

The equation that relates the acceleration of the $\mathbf{P}$ particle in frame $\mathbf{S}, \overrightarrow{\mathbf{a}}(t)=\frac{d \overrightarrow{\mathbf{v}}(t)}{d t}$, and the acceleration of

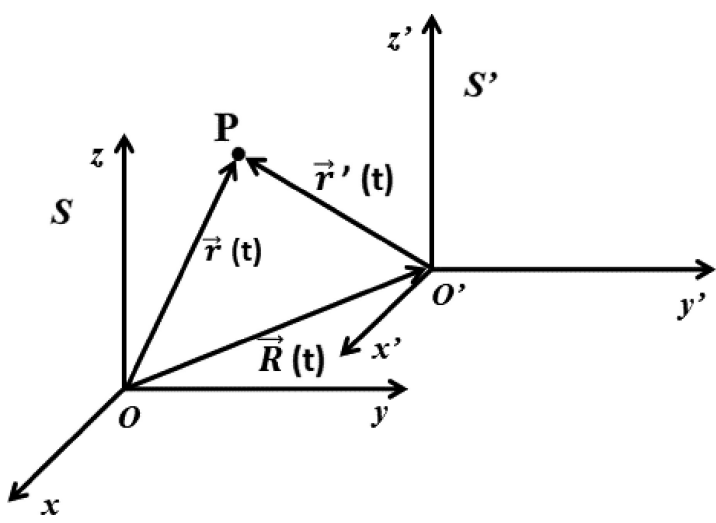

Figure 1: The position vectors of the same $P$ particle are measured from the origins $\mathbf{O}$ and $\mathbf{O}^{\prime}$ fixed in the frames $\mathbf{S}$ and $\mathbf{S}^{\prime}$, respectively. The meanings of the vectors drawn in this figure are described in the text. 
the same particle measured in frame $\mathbf{S}^{\prime}, \overrightarrow{\mathbf{a}}^{\prime}(t)=\frac{d \overrightarrow{\mathbf{v}}^{\prime}(t)}{d t}$ is derived from eq. (2),

$$
\overrightarrow{\mathbf{a}}(t)=\overrightarrow{\mathbf{a}}^{\prime}(t)+\frac{d \overrightarrow{\mathbf{V}}(t)}{d t} .
$$

If the frame $\mathbf{S}^{\prime}$ moves with constant velocity with respect to the frame $\mathbf{S}$,

$$
\frac{d \overrightarrow{\mathbf{V}}(t)}{d t}=\overrightarrow{\mathbf{0}} \Rightarrow \overrightarrow{\mathbf{a}}(t)=\overrightarrow{\mathbf{a}}^{\prime}(t)
$$

The observers at rest in each frame, $\mathbf{S}$ or $\mathbf{S}^{\prime}$, measure the same acceleration vector for the particle $\mathbf{P}$. The result (4) is independent of the equations that drive the dynamics of each particle observed from a given inertial frame.

From now on in this article we consider the physical situations where frames have constant velocity vector between them and result (4) is valid in all these frames.

Now we turn to the dynamics of moving particles with velocities much smaller than the velocity of the light. These motions are described by Newton's Second Law when they are observed from an inertial frame.

In the Classical Mechanics textbooks [10:161, 11, 15], Newton's Second Law relates the movement of the particle to the net force that acts on it,

$$
\frac{d(m \overrightarrow{\mathbf{v}}(t))}{d t}=\overrightarrow{\mathbf{F}}_{R}(t)
$$

where $\overrightarrow{\mathbf{v}}(t)$ is the velocity vector of the particle with mass $m$, and $\overrightarrow{\mathbf{F}}_{R}(t)$ is the resultant of the physical forces acting on this body. It is important to define the reference frame from which the kinematic quantities of the particle, such as: position velocity and acceleration, are measured. We call an "inertial frame" the frame where the movement of the particle is correctly described by eq. (5).

In textbooks the mass $m$ of the particle does not vary in time. We do not know, until now, any fundamental particle in Nature whose intrinsic mass is variable. It is not a settled subject if Sir Isaac Newton in his Philosophiae Naturalis Principia Mathematica leaves the possibility that in the Second Law of dynamics that the mass of the particle could not be constant [12].

As a theoretical experiment, we look in this paper for the consequences we could have if Newton's Second Law was also valid for a particle with a variable intrinsic mass $m(t)$, that is,

$$
\frac{d \overrightarrow{\mathbf{p}}(t)}{d t}=\overrightarrow{\mathbf{F}}_{R}(t),
$$

where the vector linear momentum $\overrightarrow{\mathbf{p}}(t)$ of this particle is defined as

$$
\overrightarrow{\mathbf{p}}(t) \equiv m(t) \overrightarrow{\mathbf{v}}(t)
$$

We continue to call " inertial frame the reference frame where the eq. (6a) correctly describes the motion of the massive point with variable mass $m(t) . \mathbf{S}$ is the inertial frame where eq. 6a is valid to describe the dynamics of a particle whose mass varies over time.

Let us see how eq. (6a) transforms under the Galilean transformation of the velocity (2). The frame $\mathbf{S}^{\prime}$ moves with constant velocity $\overrightarrow{\mathbf{V}}$ with respect the inertial frame S. The particle with mass $m(t)$ has velocity $\overrightarrow{\mathbf{v}}^{\prime}(t)$ for observers at rest in the frame $\mathbf{S}^{\prime}$. Substituting the equality (2) on the 1.h.s. of eq. (6a), we obtain:

$$
\frac{d \overrightarrow{\mathbf{p}}^{\prime}(t)}{d t}=-\left(\frac{d m(t)}{d t}\right) \overrightarrow{\mathbf{V}}+\overrightarrow{\mathbf{F}}_{R}(t) .
$$

$\overrightarrow{\mathbf{p}}^{\prime}(t)$ is the linear momentum vector of the particle with mass $m(t)$ measured in frame $\mathbf{S}^{\prime}$,

$$
\overrightarrow{\mathbf{p}}^{\prime}(t) \equiv m(t) \overrightarrow{\mathbf{v}}^{\prime}(t)
$$

All frames $\mathbf{S}^{\prime}$ that move with a constant velocity in relation to the inertial frame $\mathbf{S}$, is also called here an inertial frame. We will explore this point later.

If the physical forces acting on the particle with mass $m(t)$ depend on the relative position of this point body to another body, and/or the relative velocities between them, these types of forces are the same for observers at rest in any reference frame. The Galilean transformations (1) and (2) preserve relative positions and velocities between any two bodies. With this conclusion about these types of physical forces and comparing the eqs. (6a) and (7), we find that Newton's Second Law is not covariant under these transformations when applied to a particle of variable mass. Equation (7) shows that this dynamic equation depends on the velocity $\overrightarrow{\mathbf{V}}$ with which the frame $\mathbf{S}^{\prime}$ moves with respect to the inertial frame $\mathbf{S}$. We remember that we assume that Newton's Second Law $6 \mathrm{a}$ is valid in the inertial frame $\mathbf{S}$, even for particles with variable intrinsic mass.

Result (7) confirms that Newton's Second Law is not invariant under the Galilean transformations, as pointed out in ref. [8]. There would be a unique inertial frame $\mathbf{S}$ where eq. 6a describes properly the dynamics of a particle with a time-varying mass. This is certainly a very uneasy result. The equivalence of all inertial frames is the cornerstone of Classical Mechanics.

Instead of stopping here because Newton's Second Law for variable intrinsic mass particle is not covariant under Galilean transformation (2), following in this section we see what happens to Newton's three laws if eq. 6a would be valid only in the inertial frame $\mathbf{S}$.

We start by discussing Newton's third law, also called the "action and reaction law". Let us assume that there are two particles: particles 1 and $2 . \overrightarrow{\mathbf{F}}_{1 \rightarrow 2}\left(\overrightarrow{\mathbf{F}}_{2 \rightarrow 1}\right)$ is the force that particle 1 (2) acts on particle 2 (1). We presume that Newton's Third Law is valid in the inertial frame $\mathbf{S}$,

$$
\overrightarrow{\mathbf{F}}_{2 \rightarrow 1}=-\overrightarrow{\mathbf{F}}_{1 \rightarrow 2} .
$$

If the forces $\overrightarrow{\mathbf{F}}_{1 \rightarrow 2}$ and $\overrightarrow{\mathbf{F}}_{2 \rightarrow 1}$ depend on the relative position of these two particles and/or their relative 
velocities, these two vector forces are the same in all reference frames, as we argued earlier. As a consequence of this fact Newton's Third Law is valid in all reference frames, even if one or both particles has an intrinsic mass that varies over time.

In order to discuss Newton's First Law for a particle with variable intrinsic mass $m(t)$, we rewrite eq. (6a) in terms of the acceleration vector $\overrightarrow{\mathbf{a}}(t)$ of this particle measured in the inertial frame $\mathbf{S}, \overrightarrow{\mathbf{a}}(t)=\frac{\overrightarrow{\mathbf{v}}(t)}{d t}$,

$$
m(t) \overrightarrow{\mathbf{a}}(t)=-\left(\frac{d m(t)}{d t}\right) \overrightarrow{\mathbf{v}}(t)+\overrightarrow{\mathbf{F}}_{R}(t) .
$$

From eq. 10 we verify that for $\overrightarrow{\mathbf{F}}_{R}(t)=\overrightarrow{\mathbf{0}}$ Newton's First Law is not valid in the inertial frame $\mathbf{S}$ if the velocity of the particle with variable intrinsic mass $m(t)$ is not at rest during the time that the mass $m(t)$ varies in time, that is, $\overrightarrow{\mathbf{v}}(t) \neq \overrightarrow{\mathbf{0}}$.

For $\overrightarrow{\mathbf{F}}_{R}(t)=\overrightarrow{\mathbf{0}}$ and $\overrightarrow{\mathbf{v}}(t) \neq \overrightarrow{\mathbf{0}}$, throughout the movement of this particle, that is observed from the inertial frame $\mathbf{S}$, its velocity varies over time while its mass is also changing. The change in mass corresponds the inertia of the body is changing and it is also a source for varying of the velocity of the particle besides the application of a force on it.

Equation 10 gives us that the particle remains at rest on the inertial frame $\mathbf{S}$ if $\overrightarrow{\mathbf{F}}_{R}(t)=\overrightarrow{\mathbf{0}}$ at any time if the particle is initially at rest, $\overrightarrow{\mathbf{v}}(0)=\overrightarrow{\mathbf{0}}$. It does not acquire any finite velocity if at the beginning it has null velocity, even if its intrinsic mass changes.

The velocity of this same particle observed from an inertial frame $\mathbf{S}^{\prime}$ is given by eq. (2). In the case that particle with variable mass remains stationary in the inertial frame $\mathbf{S}, \overrightarrow{\mathbf{v}}(t)=\overrightarrow{\mathbf{0}}$, its velocity for observers of the inertial frame $\mathbf{S}^{\prime}$ is equal to a constant velocity, that is,

$$
\overrightarrow{\mathbf{v}}^{\prime}(t)=\overrightarrow{\mathbf{V}}
$$

where $\overrightarrow{\mathbf{V}}$ is the velocity of the frame $\mathbf{S}^{\prime}$ with respect to the frame $\mathbf{S}$. Measuring velocity $\overrightarrow{\mathbf{V}}$ would be a way to determine the relative velocity of any inertial frame $\mathbf{S}^{\prime}$ to the single inertial frame $\mathbf{S}$ where Newton's Second Law is valid.

Except in the special case of the particle of variable mass $m(t)$ remains at rest in the inertial frame $\mathbf{S}$, it is not possible to have Newton's First and Second Laws valid at same time in any inertial frame.

If we consider that Newton's Second Law (6a) is valid to describe the dynamics of a particle of mass $m(t)$ in the inertial frame $\mathbf{S}$, a possible statement for Newton's First Law modified is:

"When the net force of the physical forces applied on a particle with variable mass $m(t)$ is null, there is an inertial frame where if the body is at rest at any moment, it will remain at rest forever."

Equation (7) describes the movement of the particle with mass $m(t)$ for observers of the inertial frame $\mathbf{S}^{\prime}$, which moves with velocity $\overrightarrow{\mathbf{V}}$ with respect to the inertial frame $\mathbf{S}$. The acceleration vector of this particle in the inertial frame $\mathbf{S}^{\prime}$ is $\overrightarrow{\mathbf{a}}^{\prime}(t)=\frac{d \overrightarrow{\mathbf{v}}^{\prime}(t)}{d t}$. We rewrite eq. (7) in terms of the acceleration $\overrightarrow{\mathbf{a}}^{\prime}(t)$, that is,

$$
m(t) \overrightarrow{\mathbf{a}}^{\prime}(t)=-\left(\frac{d m(t)}{d t}\right) \underbrace{\left(\overrightarrow{\mathbf{v}}^{\prime}(t)+\overrightarrow{\mathbf{V}}\right)}_{\overrightarrow{\mathbf{v}}(t)}+\overrightarrow{\mathbf{F}}_{R}(t) .
$$

Comparing the eqs. 10 and $(12$, we verify that the dependence of the acceleration vectors of the particle with mass $m(t)$ at the frames $\mathbf{S}$ and $\mathbf{S}^{\prime}$ on the net force, that acts on it, and the velocity of the particle measured in the frame is not the same. The expression of the acceleration vector of the variable mass particle is not covariant under de Galilean transformations. But using the transformation (2) for the velocities of the particle measured in different frames, we find that the vector acceleration of the particle with mass $m(t)$ is the same in all inertial frames, retrieving the kinematic result (4). The type of motion of this variable intrinsic mass particle observed in all frames that move with constant velocity $\overrightarrow{\mathbf{V}}$ with respect to the inertial frame $\mathbf{S}$ is the same. That is the reason why we also call these frames $\mathbf{S}^{\prime}$ of inertial frames.

In Appendix A we calculate the trajectories of a particle with variable mass $m(t)$ with three special types of forces acting on it: $(i)$ the net physical force acting on the particle is null (free particle), $\overrightarrow{\mathbf{F}}_{R}(t)=\overrightarrow{\mathbf{0}} ;$ (ii) the particle is under the action of a constant force vector $\overrightarrow{\mathbf{F}}$, $\overrightarrow{\mathbf{F}}_{R}(t)=\overrightarrow{\mathbf{F}}$; and (iii) the particle of variable mass is in a free fall, $\overrightarrow{\mathbf{F}}_{R}(t)=m(t) \overrightarrow{\mathbf{g}}$, where $\overrightarrow{\mathbf{g}}$ is the acceleration due to gravity.

\subsection{Discussion on the conservation of the linear momentum vector of a particle with variable intrinsic mass}

Conservation laws are very important in solving many physical problems. They can avoid to look for the solutions of differential equations that describe the dynamics of the particles.

When the mass of the particle is constant, Newton's Second Law in any inertial frame is given by eq. (6a),

$$
\frac{d \overrightarrow{\mathbf{p}}(t)}{d t}=\overrightarrow{\mathbf{F}}_{R}(t) \quad \text { and } \quad \frac{d \overrightarrow{\mathbf{p}}^{\prime}(t)}{d t}=\overrightarrow{\mathbf{F}}_{R}(t),
$$

where $\overrightarrow{\mathbf{p}}(t)=m \overrightarrow{\mathbf{v}}(t)$ and $\overrightarrow{\mathbf{p}}^{\prime}(t)=m \overrightarrow{\mathbf{v}}^{\prime}(t)$ are the linear momentum vectors of the same particle measured in the inertial frames $\mathbf{S}$ and $\mathbf{S}^{\prime}$, respectively. From the transformation 22 we have $\overrightarrow{\mathbf{p}}(t)=\overrightarrow{\mathbf{p}}^{\prime}(t)+m \overrightarrow{\mathbf{V}}$.

When the net force $\overrightarrow{\mathbf{F}}_{R}(t)$ is null, $\overrightarrow{\mathbf{F}}_{R}(t)=\overrightarrow{\mathbf{0}}$, both eqs. (13) give the conservation of the linear momentum vectors in both inertial frames in the case of particles with constant mass,

$$
\begin{aligned}
\overrightarrow{\mathbf{p}}(t) & =\overrightarrow{\mathbf{p}}\left(t_{1}\right) \Rightarrow \overrightarrow{\mathbf{v}}(t)=\overrightarrow{\mathbf{v}}\left(t_{1}\right), \\
\overrightarrow{\mathbf{p}}^{\prime}(t) & =\overrightarrow{\mathbf{p}}^{\prime}\left(t_{1}\right) \Rightarrow \overrightarrow{\mathbf{v}}^{\prime}(t)=\overrightarrow{\mathbf{v}}^{\prime}\left(t_{1}\right)
\end{aligned}
$$


The results $14 \mathrm{a}$ and $14 \mathrm{~b}$ show that the conservation of the linear momentum vector for particles with constant mass is valid in any inertial frame if $\overrightarrow{\mathbf{F}}_{R}(t)=\overrightarrow{\mathbf{0}}$ throughout the body's movement.

Let us return to the case in which the intrinsic mass of the particle varies over time, $m(t)$. In the inertial frame $\mathbf{S}$, eq. 6a gives the equation that drives the movement of this particle,

$$
\frac{d \overrightarrow{\mathbf{p}}(t)}{d t}=\overrightarrow{\mathbf{F}}_{R}(t)
$$

with $\overrightarrow{\mathbf{p}}(t)=m(t) \overrightarrow{\mathbf{v}}(t)$. In this inertial frame when $\overrightarrow{\mathbf{F}}_{R}(t)=\overrightarrow{\mathbf{0}}$, eq. 15 gives the linear momentum vector is conserved,

$$
\overrightarrow{\mathbf{p}}(t)=\overrightarrow{\mathbf{p}}\left(t_{1}\right) \Rightarrow m(t) \overrightarrow{\mathbf{v}}(t)=m\left(t_{1}\right) \overrightarrow{\mathbf{v}}\left(t_{1}\right), \quad \forall t
$$

Unlike the case of the particle with constant mass, the conservation of the vector $\overrightarrow{\mathbf{p}}(t)$ does not imply that the velocity vector of the particle remains constant while $\overrightarrow{\mathbf{F}}_{R}(t)=\overrightarrow{\mathbf{0}}$. From eq. 16 we obtain that for particles with variable intrinsic mass the conservation of the linear momentum vector implies that its velocity vector changes its modulus depending on the variation of the mass value. This conclusion is only valid in the inertial frame $\mathbf{S}$ where Newton's Second Law 15 is valid.

Equation (7) describes the movement of the particle with mass $m(t)$ by observers at rest in the inertial frame $\mathbf{S}^{\prime}$. This frame moves with constant velocity $\overrightarrow{\mathbf{V}}$ with respect to the inertial frame $\mathbf{S}$. Since we are considering the physical situation where $\overrightarrow{\mathbf{F}}_{R}(t)=\overrightarrow{\mathbf{0}}$, in this case eq. (7) becomes

$$
\begin{aligned}
& \frac{d\left(\overrightarrow{\mathbf{p}}^{\prime}(t)+m(t) \overrightarrow{\mathbf{V}}\right)}{d t}=\overrightarrow{\mathbf{0}} \\
& \Rightarrow \underbrace{\overrightarrow{\mathbf{p}}^{\prime}\left(t_{1}\right)+m\left(t_{1}\right) \overrightarrow{\mathbf{V}}}_{\overrightarrow{\mathbf{p}}\left(t_{1}\right)}=\underbrace{\overrightarrow{\mathbf{p}}^{\prime}(t)+m(t) \overrightarrow{\mathbf{V}}}_{\overrightarrow{\mathbf{p}}(t)} \\
& \Rightarrow \overrightarrow{\mathbf{p}}^{\prime}(t)-\overrightarrow{\mathbf{p}}^{\prime}\left(t_{1}\right)=\left(m\left(t_{1}\right)-m(t)\right) \overrightarrow{\mathbf{V}}, \forall t,
\end{aligned}
$$

$t_{1}$ is any fixed time while $\overrightarrow{\mathbf{F}}_{R}(t)=\overrightarrow{\mathbf{0}}$.

During the time that the intrinsic mass $m(t)$ of the particle varies, $17 \mathrm{~b}$ shows that the linear momentum vector $\overrightarrow{\mathbf{p}}^{\prime}(t)$, measured in the inertial frame $\mathbf{S}^{\prime}$, is not conserved. From the result $17 \mathrm{a}$ we see that the vector that is conserved in the inertial frame $\mathbf{S}^{\prime}$ is the vector $\overrightarrow{\mathbf{p}}(t)$, but it is not equal to the linear momentum vector of the particle in the frame $\mathbf{S}^{\prime}$.

Only in the special frame $\mathbf{S}$, where holds Newton's Second Law 6a), the linear momentum vector is conserved when $\overrightarrow{\mathbf{F}}_{R}(t)=\overrightarrow{\mathbf{0}}$. We must be very careful to apply the conservation of the linear momentum of particles whose intrinsic mass varies over time. The nonequivalence of inertial frames, due to the dynamical description of the particles with variable intrinsic mass, breaks the idea that the conservation law should be valid in all inertial frames.

\subsection{The total mechanical energy of a particle with intrinsic mass $m(t)$}

The kinetic energy $E_{K}(t)$ of a particle is a measure of its movement in the frame in which it is observed. This quantity depends on the square of the modulus of the velocity vector of the body in the observed frame.

In inertial frames, where the motion of the particles with constant mass are followed, we have that the variation of the kinetic energy at two distinct moments, $t_{i}$, the initial time, and $t_{f}$, the final time, is equal to work realized by the net physical force acting on the particle to take it from its initial to final position [10:367],

$$
E_{K}\left(t_{f}\right)-E_{K}\left(t_{i}\right)=\int_{\substack{\overrightarrow{\mathbf{r}}\left(t_{i}\right) \\ \text { ( }}}^{\overrightarrow{\mathbf{r}}\left(t_{f}\right)} \overrightarrow{\mathbf{F}}_{R}(t) \cdot d \overrightarrow{\mathbf{r}} .
$$

$\mathcal{C}$ is the path followed by the particle to go from the initial position $\overrightarrow{\mathbf{r}}\left(t_{i}\right)$ to the final position $\overrightarrow{\mathbf{r}}\left(t_{f}\right)$.

We look for the relationship between the variation of the kinetic energy $E_{K}(t)$ and the work realized by the net force $\overrightarrow{\mathbf{F}}_{R}(t)$ acting on the particle with variable intrinsic mass to take it from its initial position up to its final position along its path $\mathcal{C}$. The work done by the net force on this particle and its kinetic energy are calculated in the inertial frame $\mathbf{S}$, where we assume that Newton's Second Law 15 is valid.

We begin extending the expression of the kinetic energy $E_{K}(t)$ to particles with variable intrinsic mass,

$$
E_{K}(t) \equiv \frac{1}{2} m(t) \overrightarrow{\mathbf{v}}(t)^{2}=\frac{\overrightarrow{\mathbf{p}}(t)^{2}}{2 m(t)} .
$$

Unlike the case of particles with constant mass, the variation of the kinetic energy $E_{K}(t)$ depends on the variation of the velocity of the particle and the change of the value of its intrinsic mass.

In what follows in this subsection, the measures of the quantities that characterize the state of movement of the particle with variable intrinsic mass $m(t)$ are performed by observers at rest in the inertial frame $\mathbf{S}$.

The time derivative of both sides of eq. 19 gives

$$
\begin{aligned}
\frac{d E_{K}(t)}{d t} & =\frac{\overrightarrow{\mathbf{p}}(t)}{m(t)} \cdot \underbrace{\left(\frac{d \overrightarrow{\mathbf{p}}(t)}{d t}\right)}_{\overrightarrow{\mathbf{F}}_{R}(t)}-E_{K}(t) \frac{d(\ln (m(t)))}{d t} \Rightarrow \\
\Rightarrow \frac{d E_{K}(t)}{d t} & =\overrightarrow{\mathbf{v}}(t) \cdot \overrightarrow{\mathbf{F}}_{R}(t)-E_{K}(t) \frac{d(\ln (m(t)))}{d t},
\end{aligned}
$$

where $\overrightarrow{\mathbf{v}}(t)$ is the velocity vector of the particle with intrinsic mass $m(t)$ measured in the inertial frame $\mathbf{S}$, and $\overrightarrow{\mathbf{F}}_{R}(t)$ is the net force that is applied to this body. To write the first term on the r.h.s. of eq. 20 we used Newton's Second Law 6a. The expression of $E_{K}(t)$ is given by eq. (19).

As in the case of particles with constant mass, the term $\overrightarrow{\mathbf{v}}(t) \cdot \overrightarrow{\mathbf{F}}_{R}(t)$ gives no contribution to the variation 


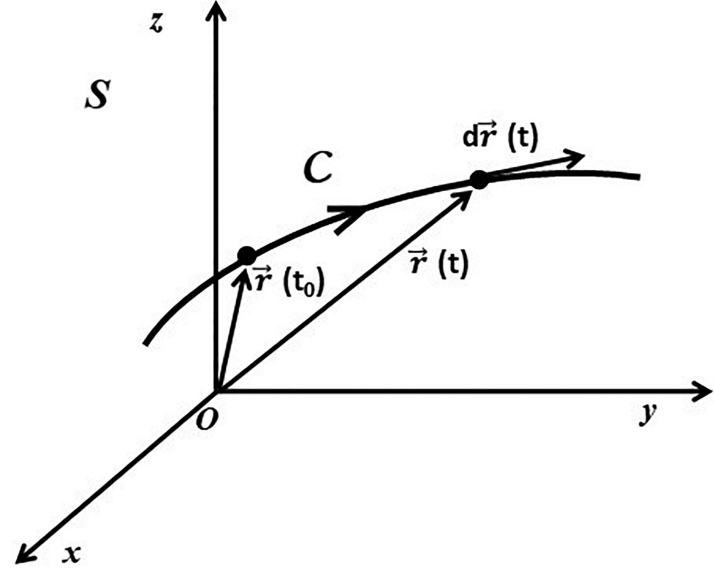

Figure 2: The path $\mathcal{C}$ is followed by the particle with intrinsic mass $m(t)$. This particle is seen by the observers fixed in the inertial frame $\mathbf{S}$. $\overrightarrow{\mathbf{r}}\left(t_{0}\right)$ and $\overrightarrow{\mathbf{r}}(t)$ are the position vectors of this particle at $t_{0}$ and $t$, respectively. $d \overrightarrow{\mathbf{r}}(t)$ is the infinitesimal displacement of the particle during the infinitesimal interval of time $t$ and $t+\Delta t$, with $\Delta t \rightarrow 0$.

of $E_{K}(t)$ if $\overrightarrow{\mathbf{F}}_{R}(t)$ is perpendicular to the velocity of the particle in the inertial frame $\mathbf{S}$.

The term $-E_{K}(t) \frac{d(\ln (m(t)))}{d t}$ on the r.h.s. of eq. 20 gives a negative (positive) contribution to the variation of the kinetic energy when the mass of the particle increases (diminishes). The increase (diminution) of the mass of the particle increase (decrease) its inertial that decreases (enlarge) its velocity.

Remembering that the velocity $\overrightarrow{\mathbf{v}}(t)$ can be written in terms of the infinitesimal displacement $d \overrightarrow{\mathbf{r}}(t)$ along the path $\mathcal{C}$ followed by the particle, see Fig. 2 .

$$
\overrightarrow{\mathbf{v}}(t)=\frac{d \overrightarrow{\mathbf{r}}(t)}{d t}
$$

and the result 20 is rewritten as

$$
d E_{K}(t)=d \overrightarrow{\mathbf{r}}(t) \cdot \overrightarrow{\mathbf{F}}_{R}(t)-E_{K}(t) d(\ln (m(t))) .
$$

The first term $d \overrightarrow{\mathbf{r}}(t) \cdot \overrightarrow{\mathbf{F}}_{R}(t)$ on the r.h.s. of eq. 22 is the infinitesimal work done by the net force $\overrightarrow{\mathbf{F}}_{R}(t)$ on the particle with variable mass along its infinitesimal displacement $d \overrightarrow{\mathbf{r}}(t)$.

The integral form of result 22 is

$$
\begin{aligned}
& E_{K}(t)-E_{K}\left(t_{0}\right)
\end{aligned}
$$

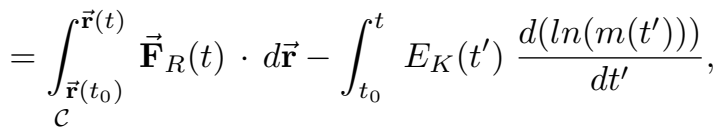

showing that for particles with intrinsic mass $m(t)$ you have two sources for varying their kinetic energy $E_{K}(t)$ : $(i)$ the work of the net physical force $\overrightarrow{\mathbf{F}}_{R}(t)$ that acts on the particle; (ii) the variation of the inertia of the particle.

As we said before, the dependence of $E_{K}(t)$ on the mass $m(t)$ of the particle, see definition $(19)$, is responsible for part of the variation of this kinematic function, but the velocity $\overrightarrow{\mathbf{v}}(t)$ also changes with the the variation of body mass, see eq. (10). For distinguish these two contributions to the variation of $E_{K}(t)$, we rewrite eq. 10 introducing a tracer $a$, that is,

$$
m(t) \overrightarrow{\mathbf{a}}(t)=-a\left(\frac{d m(t)}{d t}\right) \overrightarrow{\mathbf{v}}(t)+\overrightarrow{\mathbf{F}}_{R}(t) .
$$

At the end of the calculation of $\frac{d E_{K}(t)}{d t}$ we take $a=1$. The presence of the tracer $a$ in the expression $\frac{d E_{K}(t)}{d t}$ permits us to know the contribution of the first term on the r.h.s. of eq. (24) for the variation of the kinetic energy, $E_{K}(t)$.

We write $E_{K}(t)$ as

$$
E_{K}(t)=\frac{1}{2} m(t) \overrightarrow{\mathbf{v}}(t)^{2},
$$

whose the time derivative on both sides gives

$$
\begin{aligned}
\frac{d E_{K}(t)}{d t}= & m(t) \overrightarrow{\mathbf{v}}(t) \cdot \frac{d \overrightarrow{\mathbf{v}}(t)}{d t} \\
& +\frac{1}{2} \frac{d(m(t))}{d t} \overrightarrow{\mathbf{v}}(t) \cdot \overrightarrow{\mathbf{v}}(t) \Rightarrow \\
\underbrace{\Rightarrow}_{(24)} \frac{d E_{K}(t)}{d t}= & \overrightarrow{\mathbf{v}}(t) \cdot \overrightarrow{\mathbf{F}}_{R}(t) \\
& -(2 a-1) E_{K}(t)\left(\frac{d(\ln (m(t)))}{d t}\right) .
\end{aligned}
$$

If we take $a=1$ on the r.h.s. of eq. 26 , we recover the result 20. The previous result permits to have a clear understanding of the two sources to the term $-E_{K}(t)\left(\frac{d(\ln (m(t)))}{d t}\right)$ in the time variation of the kinetic energy $E_{K}(t)$.

So far, the net physical force $\overrightarrow{\mathbf{F}}_{R}(t)$ could be any force. Now, we restrict our discussion to the set of conservative forces,

$$
\overrightarrow{\mathbf{F}}_{R}(t)=-\vec{\nabla} V(\overrightarrow{\mathbf{r}})
$$

$V(\overrightarrow{\mathbf{r}})$ is a potential energy. This function has no explicit dependence on the time $t$.

Substituting equality 27 on the r.h.s. of eq. (22), it becomes

$$
\begin{aligned}
d E_{K}(t) & =-d V(\overrightarrow{\mathbf{r}})-E_{K}(t) d(\ln (m(t))) \Rightarrow \\
\Rightarrow d\left[E_{K}(t)+V(\overrightarrow{\mathbf{r}})\right] & =-E_{K}(t) d(\ln (m(t))) .
\end{aligned}
$$

Using the usual definition for total mechanical energy to define it for a particle with variable intrinsic mass, $E_{T}(t)$,

$$
E_{T}(t) \equiv E_{K}(t)+V(\overrightarrow{\mathbf{r}}),
$$

eq. 28 becomes

$$
d\left(E_{T}(t)\right)=-E_{K}(t) d(\ln (m(t))),
$$


that has the integral form,

$$
E_{T}(t)-E_{T}\left(t_{0}\right)=-\int_{t_{0}}^{t} E_{K}\left(t^{\prime}\right) \frac{d\left(\ln \left(m\left(t^{\prime}\right)\right)\right)}{d t^{\prime}} d t^{\prime}
$$

The results 30a and 30b show that the total mechanical energy of the particle with variable intrinsic mass, $E_{T}(t)$, under the action of a conservative force, is not constant while the intrinsic mass of the particle varies over time. The integral on the r.h.s. of eq. $30 \mathrm{~b}$ depends on the path followed by particle starting from its initial position $\overrightarrow{\mathbf{r}}\left(t_{0}\right)$ to the position of the particle at time $t, \overrightarrow{\mathbf{r}}(t)$. This result is completely different from the physical situation when the mass of the particle is constant.

As a final reminder, the results obtained in this subsection on mechanical energy of a particle with variable intrinsic mass are valid in the inertial frame $\mathbf{S}$, where Newton's Second Law 15 is valid.

\section{Newton's Second Law for an extended body with variable number of particles}

Thomaz and Corrêa Silva studied in reference [9] the dynamics of the center of mass (CM) of a system of particles whose total mass varies during an interval of time. They considered two mechanisms for the mass of the CM to change over time: $(i)$ all particles in the set have constant mass, but one of the particles leaves the system; (ii) the mass of the one of the particles, which belong to the set, varies with time.

In this section we discuss the equation of motion of a set of particles with constant mass whose number of members varies of one unit. To simplify the presentation of this matter we assume that at the beginning of the system, called by $\mathcal{A}$, has two particles, $N=2$, and in the end it has only one particle. This case corresponds to have $N=2$ in the eqs. (60) and (61) of the reference 9 . This is a oversimplified description of systems with variable mass, such as rockets, conveyors for mineral deposits and so on.

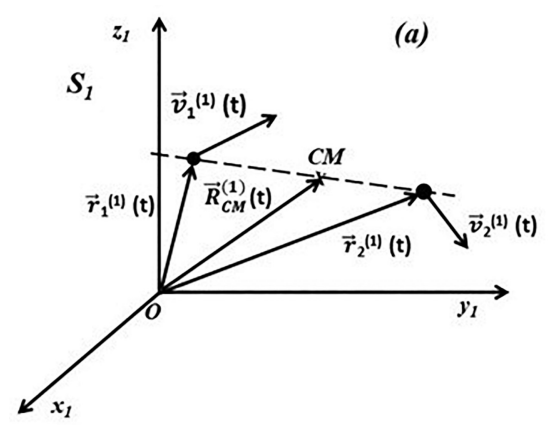

The particles 1 and 2 have constant masses $m_{1}$ and $m_{2}$, respectively. In any inertial frame their motions are described by Newton's Second Law (6a). In an inertial frame $\mathcal{S}_{1}$, the equations that driven the motions of the two particles in the set $\mathcal{A}$ are:

$$
\begin{aligned}
& \frac{d \overrightarrow{\mathbf{p}}_{1}^{(1)}(t)}{d t}=\frac{d\left(m_{1} \overrightarrow{\mathbf{v}}_{1}^{(1)}(t)\right)}{d t}=\overrightarrow{\mathbf{F}}_{1}^{(e x t)}(t)+\overrightarrow{\mathbf{F}}_{2 \rightarrow 1}^{(i n t)}(t), \\
& \frac{d \overrightarrow{\mathbf{p}}_{2}^{(1)}(t)}{d t}=\frac{d\left(m_{2} \overrightarrow{\mathbf{v}}_{2}^{(1)}(t)\right)}{d t}=\overrightarrow{\mathbf{F}}_{2}^{(e x t)}(t)+\overrightarrow{\mathbf{F}}_{1 \rightarrow 2}^{(i n t)}(t) .
\end{aligned}
$$

$\overrightarrow{\mathbf{v}}_{i}^{(1)}(t)$ is the velocity of the $i-t h$ particle, with $i=1,2$, measured by observers at rest in the inertial frame $\mathbf{S}_{1}$. $\overrightarrow{\mathbf{F}}_{i}^{(e x t)}(t)$ is the net external physical force applied to the $i$ - th particle, with $i=1,2$, and $\overrightarrow{\mathbf{F}}_{2 \rightarrow 1}^{(i n t)}(t)\left(\overrightarrow{\mathbf{F}}_{1 \rightarrow 2}^{(i n t)}(t)\right)$ is the internal force that particle $2(1)$ acts on the particle 1 (2). It is important to point out that $\overrightarrow{\mathbf{F}}_{2 \rightarrow 1}^{(i n t)}(t)$ and $\overrightarrow{\mathbf{F}}_{1 \rightarrow 2}^{(i n t)}(t)$ are internal forces while both particles belong to the set $\mathcal{A}$ of particles.

The position of the CM of the system $\mathcal{A}$ in the inertial frame $\mathbf{S}_{1}, \overrightarrow{\mathbf{R}}_{C M}^{(1)}(t)$, is

$$
\overrightarrow{\mathbf{R}}_{C M}^{(1)}(t) \equiv \frac{m_{1} \overrightarrow{\mathbf{r}}_{1}^{(1)}(t)+f(-t) m_{2} \overrightarrow{\mathbf{r}}_{2}^{(1)}(t)}{m_{1}+f(-t) m_{2}} .
$$

We plot in Fig. 3 the positions and velocities of particles 1 and 2, $\left.\overrightarrow{\mathbf{r}}_{1}^{(1)}(t), \overrightarrow{\mathbf{v}}_{1}^{(1)}(t)\right)$ and $\left(\overrightarrow{\mathbf{r}}_{2}^{(1)}(t), \overrightarrow{\mathbf{v}}_{2}^{(1)}(t)\right)$, respectively, in an inertial $\mathbf{S}_{1}$. The position $\overrightarrow{\mathbf{R}}_{C M}^{(1)}(t)$ is also drawn in this figure.

We associate an imaginary particle to the movement of the CM of the set $\mathcal{A}$. The position of this imaginary particle coincides with the location of the CM of the set $\mathcal{A}$ at all times. The mass of this imaginary particle, $M_{C M}(t)$, is chosen to be equal to

$$
M_{C M}(t) \equiv m_{1}+f(-t) m_{2} .
$$

We follow reference [9], so that the function $f(t)$ is defined as being

$$
f(t) \equiv\left\{\begin{array}{l}
0, \quad t<-\frac{1}{\alpha} \\
g(t), \quad-\frac{1}{\alpha} \leq t_{1} \geq \frac{1}{\alpha} . \\
1, \quad t>\frac{1}{\alpha}
\end{array}\right.
$$

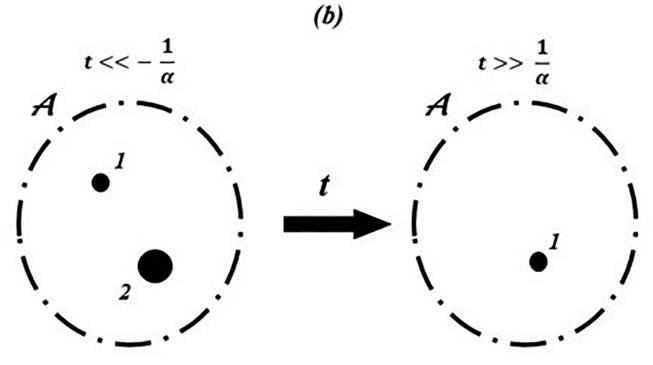

Figure 3: $(a)$ The position and the velocity vectors of particle 1 (2) for observers at rest in the inertial frame $S_{1}, \overrightarrow{\mathbf{r}}_{1}(t)\left(\overrightarrow{\mathbf{r}}_{2}(t)\right)$ and $\overrightarrow{\mathbf{v}}_{1}(t)\left(\overrightarrow{\mathbf{v}}_{2}(t)\right)$. We also draw the CM position vector of this set of particles, $\overrightarrow{\mathbf{R}}_{C M}(t) ;(b)$. We draw the set $\mathcal{A}$ with the particles that belong to it, for $t \ll \frac{-1}{\alpha}$ and $t \gg \frac{1}{\alpha}$, when we have a well-defined number of particles in the system $\mathcal{A}$. 
The $g(t)$ function smoothly connects the two regions of $f(t)$ with $t<-\frac{1}{\alpha}$ and $t>\frac{1}{\alpha}$. In the definitions 32 and (33), along with the function (34), describe a physical situation where particle 2 leaves the set $\mathcal{A}$ for $t>\frac{1}{\alpha}{ }^{1}$ In Fig. $3 \mathrm{p}$ we show the particles that are part of the system $\mathcal{A}$ for the time regions $t \ll-\frac{1}{\alpha}$ and $t \gg \frac{1}{\alpha}$, when the number of particles in this set is well defined. Note that the variation of the value of the mass of the CM, $M_{C M}(t)$, comes from the fact that the number of particles in the set $\mathcal{A}$ varies over time. This is a different framework that we studied in section 2 .

The velocity of the $\mathrm{CM}$ of the system $\mathcal{A}$ in an inertial frame $\mathbf{S}_{1}$ is

$$
\begin{aligned}
\overrightarrow{\mathbf{V}}_{C M}^{(1)}(t)= & \frac{d \overrightarrow{\mathbf{R}}_{C M}^{(1)}(t)}{d t} \\
= & \frac{m_{1} \overrightarrow{\mathbf{v}}_{1}^{(1)}(t)+f(-t) m_{2} \overrightarrow{\mathbf{v}}_{2}^{(1)}(t)}{M_{C M}(t)}+ \\
& +\frac{1}{M_{C M}(t)}\left(\frac{d M_{C M}(t)}{d t}\right)\left(\overrightarrow{\mathbf{r}}_{2}^{(1)}(t)-\overrightarrow{\mathbf{R}}_{C M}^{(1)}(t)\right) .
\end{aligned}
$$

The linear momentum vector of the $\mathrm{CM}$ of the set $\mathcal{A}$ in an inertial frame $\mathbf{S}_{\mathbf{1}}, \overrightarrow{\mathbf{P}}_{C M}^{(1)}(t)$, is defined in the same way as we define for any punctual body,

$$
\overrightarrow{\mathbf{P}}_{C M}^{(1)}(t) \equiv M_{C M}(t) \overrightarrow{\mathbf{V}}_{C M}^{(1)}(t)
$$

From eq. $35 \mathrm{~b}$ we obtain the expression of the linear momentum of the $\mathrm{CM}$ of the set $\mathcal{A}$ of particles in terms of the physical quantities of the particles that belong to it. All vectors are measured in an inertial frame $\mathbf{S}_{1}$,

$$
\begin{aligned}
\overrightarrow{\mathbf{P}}_{C M}^{(1)}(t)= & m_{1} \overrightarrow{\mathbf{v}}_{1}^{(1)}(t)+f(-t) m_{2} \overrightarrow{\mathbf{v}}_{2}^{(1)}(t) \\
& +\left(\frac{d M_{C M}(t)}{d t}\right)\left(\overrightarrow{\mathbf{r}}_{2}^{(1)}(t)-\overrightarrow{\mathbf{R}}_{C M}^{(1)}(t)\right)
\end{aligned}
$$

The previous expression of $\overrightarrow{\mathbf{P}}_{C M}^{(1)}(t)$ is reduced to the known linear momentum vector of the CM presented in textbooks when $\frac{d M_{C M}(t)}{d t}=0$, and $t<-\frac{1}{\alpha}$ or $t>\frac{1}{\alpha}$.

Newton's Second Law 31a and 31b of physical particles 1 and 2, valid in any inertial frame, permits calculating the equation of motion of the $\mathrm{CM}$ of the set $\mathcal{A}$, and it is equal to

$$
\begin{aligned}
\frac{d \overrightarrow{\mathbf{P}}_{C M}^{(1)}(t)}{d t}= & \overrightarrow{\mathbf{F}}_{1}^{(e x t)}(t)+\overrightarrow{\mathbf{F}}_{2 \rightarrow 1}^{(i n t)}(t) \\
& +f(-t)\left[\overrightarrow{\mathbf{F}}_{2}^{(e x t)}(t)+\overrightarrow{\mathbf{F}}_{1 \rightarrow 2}^{(i n t)}(t)\right]+
\end{aligned}
$$

${ }^{1}$ All the arguments presented in section 3 are still valid if initially the set $\mathcal{A}$ has only the particle 1 and after $t>\frac{1}{\alpha}$ the particle 2 is included in the set $\mathcal{A}$. To implement this scenario we could have the function $f_{c}(t)$,

$$
f_{c}(t) \equiv \begin{cases}1, & t<-\frac{1}{\alpha} \\ g_{c}(t), & -\frac{1}{\alpha} \leq t \leq \frac{1}{\alpha} . \\ 0, & t \geq \frac{1}{\alpha}\end{cases}
$$

The function $g_{c}(t)$ must connect the values of the $f_{c}(t)$ function in the regions $t<-\frac{1}{\alpha}$ and $t \geq \frac{1}{\alpha}$.

$$
\begin{aligned}
& +\frac{d}{d t}\left[\left(\frac{d M_{C M}(t)}{d t}\right)\left(\overrightarrow{\mathbf{r}}_{2}^{(1)}(t)-\overrightarrow{\mathbf{R}}_{C M}^{(1)}(t)\right)\right]+ \\
& +\left(\frac{d M_{C M}(t)}{d t}\right) \overrightarrow{\mathbf{v}}_{2}^{(1)}(t) .
\end{aligned}
$$

The last term on the r.h.s. of eq. 37) depends on the velocity on the particle 2 measured in the inertial frame $\mathbf{S}_{1}$. Is this equation of motion of the CM of the system $\mathcal{A}$ invariant under Galilean transformations (1) and $(2)$ ?

Let $\mathbf{S}_{2}$ be another inertial frame. The relations between the position and velocity vectors measured in the inertial frames $\mathbf{S}_{1}$ and $\mathbf{S}_{2}$ are

$$
\begin{aligned}
\overrightarrow{\mathbf{R}}_{C M}^{(1)}(t) & =\overrightarrow{\mathbf{R}}_{C M}^{(2)}(t)+\overrightarrow{\mathbf{V}} t, \\
\overrightarrow{\mathbf{V}}_{C M}^{(1)}(t) & =\overrightarrow{\mathbf{V}}_{C M}^{(2)}(t)+\overrightarrow{\mathbf{V}}, \\
\overrightarrow{\mathbf{r}}_{2}^{(1)}(t) & =\overrightarrow{\mathbf{r}}_{2}^{(2)}(t)+\overrightarrow{\mathbf{V}} t, \\
\overrightarrow{\mathbf{v}}_{2}^{(1)}(t) & =\overrightarrow{\mathbf{v}}_{2}^{(2)}(t)+\overrightarrow{\mathbf{V}} .
\end{aligned}
$$

$\overrightarrow{\mathbf{V}}$ is the constant velocity of the inertial frame $\mathbf{S}_{2}$ with respect to the inertial frame $\mathbf{S}_{1} \cdot \overrightarrow{\mathbf{R}}_{C M}^{(i)}(t)$ and $\overrightarrow{\mathbf{V}}_{C M}(t)^{(i)}(t)$ are the position and velocity vectors of the $\mathrm{CM}$ of the set $\mathcal{A}$ in the inertial frame $\mathbf{S}_{i}, i=1$ e 2 . The position and the velocity vectors of particle 2 measured by observers at rest in the inertial frame $\mathbf{S}_{i}$ are $\overrightarrow{\mathbf{r}}_{2}^{(i)}(t)$ and $\overrightarrow{\mathbf{v}}_{2}^{(i)}(t)$, with $i=1,2$.

From the relations 38a-38d we obtain that the relative position of the particle 2 with respect to the position of the $\mathrm{CM}$ of the set $\mathcal{A}$ is the same in all inertial frame: $\overrightarrow{\mathbf{r}}_{2}^{(1)}(t)-\overrightarrow{\mathbf{R}}_{C M}^{(1)}(t)=\overrightarrow{\mathbf{r}}_{2}^{(2)}(t)-\overrightarrow{\mathbf{R}}_{C M}^{(2)}(t)$.

We use the relations $38 \mathrm{a}-(38 \mathrm{~d})$ again to rewrite the eq. (37) in terms of the vectors measured in the inertial frame $\mathbf{S}_{2}$. After a few algebraic manipulations the eq. 37 gives:

$$
\begin{aligned}
& \frac{d \overrightarrow{\mathbf{P}}_{C M}^{(2)}(t)}{d t} \\
& =\overrightarrow{\mathbf{F}}_{1}^{(e x t)}(t)+\overrightarrow{\mathbf{F}}_{2 \rightarrow 1}^{(i n t)}(t)+f(-t)\left[\overrightarrow{\mathbf{F}}_{2}^{(e x t)}(t)+\overrightarrow{\mathbf{F}}_{1 \rightarrow 2}^{(i n t)}(t)\right]+ \\
& \quad+\frac{d}{d t}\left[\left(\frac{d M_{C M}(t)}{d t}\right)\left(\overrightarrow{\mathbf{r}}_{2}^{(2)}(t)-\overrightarrow{\mathbf{R}}_{C M}^{(2)}(t)\right)\right]+ \\
& \quad+\underbrace{\left(\frac{d M_{C M}(t)}{d t}\right)\left(\overrightarrow{\mathbf{v}}_{2}^{(2)}(t)+\overrightarrow{\mathbf{V}}\right)-\left(\frac{d M_{C M}(t)}{d t}\right) \overrightarrow{\mathbf{V}}}_{\left(\frac{d M_{C M}(t)}{d t}\right) \overrightarrow{\mathbf{v}}_{2}^{(2)}(t)}
\end{aligned}
$$

Equation (39) shows that eq. (37), that describes the movement of the $\mathrm{CM}$ of the set $\mathcal{A}$ in the inertial frame $\mathbf{S}_{1}$ is invariant under Galilean transformations (1) and (2) for all inertial frames, although it explicitly depends on the velocity of the particle leaving the system. This result is different from the one that we discussed in the section 2, where we assumed that the intrinsic mass of the elementary particle change over time. Remember that Newton's Second Law (15) of a 
particle with variable intrinsic mass is not invariant under the Galilean transformations that connects the measurements of kinetic quantities of particles realized in inertial frames.

We return to the eqs. (37) and (39) to discuss the conservation law satisfied by the motion of the CM of the set $\mathcal{A}$ when this system is isolated and the physical forces satisfy the conditions:

$$
\overrightarrow{\mathbf{F}}_{1}^{(e x t)}(t)+\overrightarrow{\mathbf{F}}_{2 \rightarrow 1}^{(i n t)}(t)=\overrightarrow{\mathbf{0}}
$$

and

$$
\overrightarrow{\mathbf{F}}_{2}^{(e x t)}(t)+\overrightarrow{\mathbf{F}}_{1 \rightarrow 2}^{(i n t)}(t)=\overrightarrow{\mathbf{0}}
$$

Under the conditions 40a and 40b, the eq. (37) in any inertial frame is equal to

$$
\begin{gathered}
\frac{d}{d t}\left[\overrightarrow{\mathbf{P}}_{C M}(t)-\left(\frac{d M_{C M}(t)}{d t}\right)\left(\overrightarrow{\mathbf{r}}_{2}(t)-\overrightarrow{\mathbf{R}}_{C M}(t)\right)\right. \\
\left.-M_{C M}(t) \overrightarrow{\mathbf{v}}_{2}(t)\right]=\overrightarrow{\mathbf{0}}
\end{gathered}
$$

In eq. (41), we removed the top indexes on all vectors since this equation is valid in any inertial frame.

The conditions 40a and 40b include the possibility of the particles being free, that is, $\overrightarrow{\mathbf{F}}_{1}^{(e x t)}(t)=\overrightarrow{\mathbf{F}}_{2 \rightarrow 1}^{(i n t)}(t)=$ $\overrightarrow{\mathbf{F}}_{2}^{(e x t)}(t)=\overrightarrow{\mathbf{F}}_{1 \rightarrow 2}^{(i n t)}(t)=\overrightarrow{\mathbf{0}}$. From the result 411 we find that in this case the linear momentum vector of the $\mathrm{CM}$ of the set $\mathcal{A}, \overrightarrow{\mathbf{P}}_{C M}(t)$, is not conserved during the time interval in which the value of its mass $M_{C M}(t)$ is changing. During the time interval in which particle is leaving the set $\mathcal{A}, t \in\left(-\frac{1}{\alpha}, \frac{1}{\alpha}\right)$, the vector that conserves is:

$$
\begin{gathered}
\overrightarrow{\mathbf{P}}_{C M}(t)-\left(\frac{d M_{C M}(t)}{d t}\right)\left(\overrightarrow{\mathbf{r}}_{2}(t)-\overrightarrow{\mathbf{R}}_{C M}(t)\right) \\
-M_{C M}(t) \overrightarrow{\mathbf{v}}_{2}(t)=\text { constant vector. }
\end{gathered}
$$

This vector is also conserved during the whole movement of the set $\mathcal{A}$ of particles. As in the case of the particle with variable intrinsic mass, discussed in the section 2 , the linear momentum vector of the CM of the set with two free particles, an isolated system, is not conserved during the time that one of the masses is leaving the system.

To exemplify the result 42 we follow the reference 9 and we assume that the function $f(t)$ is equal to:

$$
f(t)=\frac{1}{2}(1+\tanh (\alpha t))
$$

with $\alpha>0$. The masses of the two particles in the set $\mathcal{A}$ are: $m_{1}=m_{0}$ and $m_{2}=2 m_{0}$.

In this case, the mass 33 of the $\mathrm{CM}$ of the set $\mathcal{A}$ is

$$
\begin{aligned}
M_{C M}(t) & =m_{0}+2 m_{0} \times f(-t) \\
& =m_{0}(2-\tanh (\alpha t)) .
\end{aligned}
$$

Substituting the conditions 40a and 40b on the r.h.s. of the eqs. 31a and 31b, respectively, we obtain, in any inertial frame,

$$
\overrightarrow{\mathbf{v}}_{1}(t)=\overrightarrow{\mathbf{v}}_{1} \quad \text { and } \quad \overrightarrow{\mathbf{v}}_{2}(t)=\overrightarrow{\mathbf{v}}_{2},
$$

where $\overrightarrow{\mathbf{v}}_{1}$ and $\overrightarrow{\mathbf{v}}_{2}$ are constant vectors. We also have

$$
\begin{aligned}
& \overrightarrow{\mathbf{r}}_{1}(t)=\overrightarrow{\mathbf{r}}_{1}(0)+\overrightarrow{\mathbf{v}}_{1} t, \\
& \overrightarrow{\mathbf{r}}_{2}(t)=\overrightarrow{\mathbf{r}}_{2}(0)+\overrightarrow{\mathbf{v}}_{2} t .
\end{aligned}
$$

$\overrightarrow{\mathbf{r}}_{1}(0)$ and $\overrightarrow{\mathbf{r}}_{2}(0)$ are the position vectors of the particles 1 and 2 respectively at $t=0$.

Equation $35 \mathrm{~b}$ gives the relationship between the velocity of the CM of the set $\mathcal{A}, \overrightarrow{\mathbf{V}}_{C M}(t)$, and the informations of the particles that belong to this system. Since $\overrightarrow{\mathbf{P}}_{C M}(t)=M_{C M}(t) \overrightarrow{\mathbf{V}}_{C M}(t)$, for this example of two free particles, with particle 2 leaving the system after $t \gtrsim-\frac{1}{\alpha}$, as described by function $f(t)$ given by (43). The linear momentum vector in any inertial frame, $\overrightarrow{\mathbf{P}}_{C M}(t)$, is

$$
\begin{aligned}
\overrightarrow{\mathbf{P}}_{C M}(t)= & m_{0} \overrightarrow{\mathbf{v}}_{1}+m_{0}(1-\tanh (\alpha t)) \overrightarrow{\mathbf{v}}_{2} \\
& -m_{0}\left(\frac{\alpha \operatorname{sech}^{2}(\alpha t)}{2-\tanh (\alpha t)}\right) \\
& \times\left[\overrightarrow{\mathbf{r}}_{1}(0)-\overrightarrow{\mathbf{r}}_{2}(0)+\left(\overrightarrow{\mathbf{v}}_{1}-\overrightarrow{\mathbf{v}}_{2}\right) t\right]
\end{aligned}
$$

with $\alpha>0$.

For $t \ll-\frac{1}{\alpha}$, we have

$$
\overrightarrow{\mathbf{P}}_{C M}^{(b)} \approx m_{0} \overrightarrow{\mathbf{v}}_{1}+2 m_{0} \overrightarrow{\mathbf{v}}_{2}
$$

For $t \gg+\frac{1}{\alpha}$ we obtain

$$
\overrightarrow{\mathbf{P}}_{C M}^{(a)} \approx m_{0} \overrightarrow{\mathbf{v}}_{1}
$$

The vector $\overrightarrow{\mathbf{P}}_{C M}(t)$ is constant outside of the time interval $\left(-\frac{1}{\alpha}, \frac{1}{\alpha}\right)$ while the value of the mass of the CM of the set $\mathcal{A}$ is changing. Although the vectors $\overrightarrow{\mathbf{P}}_{C M}^{(b)}$ and $\overrightarrow{\mathbf{P}}_{C M}^{(a)}$ are constant vectors, they are different vectors.

As an example of these results, we consider that the two free particles perform a one-dimensional movement along the $x$-axis. The particles satisfy the following initial conditions:

$$
\begin{gathered}
\overrightarrow{\mathbf{r}}_{1}(0)=\overrightarrow{\mathbf{r}}_{2}(0), \\
\overrightarrow{\mathbf{v}}_{1}=1 \hat{\imath} \quad \text { and } \quad \overrightarrow{\mathbf{v}}_{2}=-3 \hat{\imath} .
\end{gathered}
$$

In this case, the vector $\overrightarrow{\mathbf{P}}_{C M}(t)=P_{C M}(t) \hat{\imath}$, where $\hat{\imath}$ is the unit vector along the $x$-axis. Then,

$$
\frac{P_{C M}(t)}{m_{0}}=1-3 \cdot(1-\tanh (\alpha t))-\frac{4 \alpha \operatorname{tsech}^{2}(\alpha t)}{2-\tanh (\alpha t)} \text {. }
$$

We plot in Fig. 4 the graph $\frac{P_{C M}(t)}{m_{0}} \times t$ with $\alpha=8$. In this simple example we verify that the linear momentum of the CM of a system with a variable number of particles does not conserve during the entire motion of 


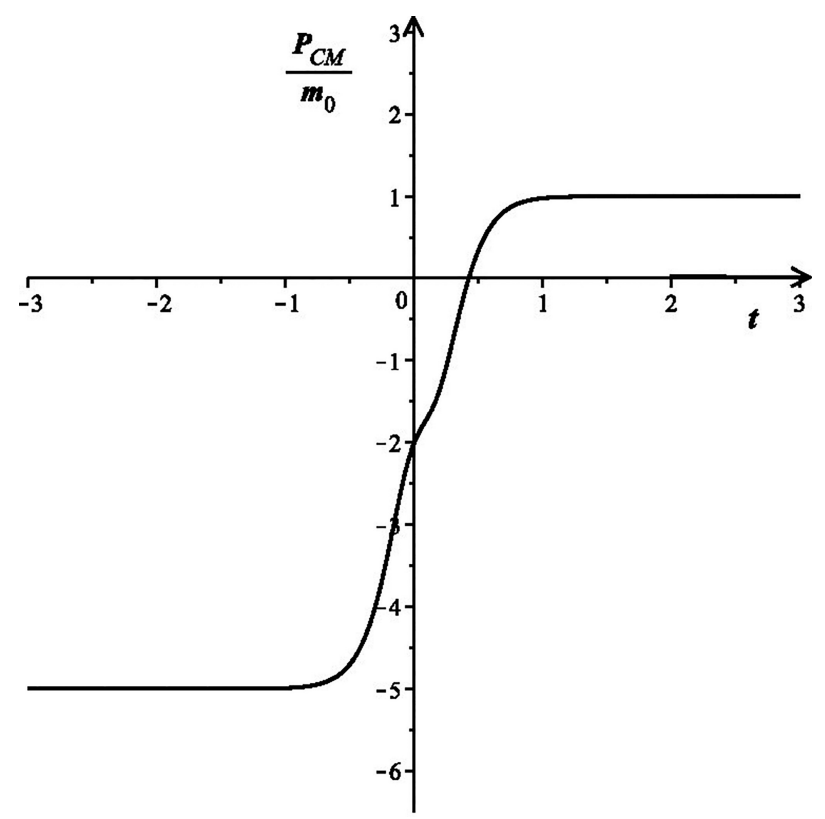

Figure 4: Plot of the expression 50 of the function $\frac{P_{C M}(t)}{m_{0}}$, with $\alpha=8$. We have $\frac{P_{C M}}{m_{0}} \approx-5$ for $t \lesssim-1$ and $\frac{P_{C M}}{m_{0}} \approx 1$ for $t \gtrsim 1$. During the interval $t \in(-1,1)$, when the particle 2 is no longer part of the set $\mathcal{A}, P_{C M}(t)$ is not a conserved quantity.

the CM of the set of particles. The value of $P_{C M}(t)$ before and after the time interval in which its mass varies are constant but the values of theses constants are distinct. The value of the $P_{C M}(t)$ varies during the time in which the particle 2 is leaving the system. We must be careful when applying the conservation of the linear momentum for the movement of the CM of mass of a system with variable mass.

The results of section 3 obtained for the $\mathrm{CM}$ of a set of particles, that we has called set $\mathcal{A}$, can be easily extended to the case of a system with $N$ particles, $N \geq 2$, with all $N$ particles having constant mass, where one or more particles leave or enter in this system during the time we follow the motion of the CM from an inertial frame.

\section{Conclusions}

The fundamental particles that we know today in Nature have constant mass. But in Physics textbooks, the Newton's Second Law that describes the motion of particles followed by observers at rest in inertial frames, relates the time variation of their linear momentum vectors with the vector resulting from the physical forces acting on them. When we mention a body with a variable mass, composite systems like rockets, currents hitting the ground, and so on, come to mind. These bodies are made of parts, with constant mass value, that can leave or be added to the system that we follow, changing the value of their total mass over time. We have another theoretical possibility: a particle whose value of its intrinsic mass varies with time.
It is possible that Newton's Second Law 15 distinguishes the dynamics of an intrinsically variable particle mass from a complex body formed by parts, with constant mass, that break or add to the body under study, changing its value?

In section 2 of this paper we assume that the particles has a variable intrinsic mass and that the Newton's Second Law is valid in one particular inertial frame, that we call $\mathbf{S}$ inertial frame. In this section we retrieve the known result that this Newton's Law is not covariant under the Galilean transformations during the time interval that mass of the particle varies with time. We explore the consequences of the non-covariance of the equation that drives the particle dynamics observed from different inertial frames. Newton's Third Law is still valid in all frames, but the Newton's First Law is not valid in any inertial frame as the mass of the particle varies. If the mass of the particle varies over a finite interval of time, the Newton's Second Law remains valid in all inertial frames from where the body is observed while its mass is kept constant. But this Newton's Law is only valid in one inertial frame, which we call the inertial frame $\mathbf{S}$, during the interval of time that the mass of the particle vary. We have no recipe for determining this unique inertial frame where Newton's Second Law is valid to describe the motion of the particle with variable intrinsic mass. The equivalence of all inertial references under the Galilean transformations are lost while the intrinsic mass of the particles varies.

One of the consequences of the loss of the equivalence of all inertial frames is that the conservation of the linear momentum vector of the particle with variable intrinsic mass in the absence of a net physical force acting on it, that is, $\overrightarrow{\mathbf{F}}_{R}(t)=\overrightarrow{\mathbf{0}}$, is just valid in the particular inertial frame $\mathbf{S}$, where Newton's Second Law 15 is valid. When $\overrightarrow{\mathbf{F}}_{R}(t)=\overrightarrow{\mathbf{0}}$, the body with variable intrinsic mass has an accelerated or slow movement while its mass varies, unless it is at rest in the $\mathbf{S}$ inertial frame. The variation of the velocity of the particle comes from the change of the value of its inertia.

We also present how changing the value of its intrinsic mass affects its mechanical energy. We show that the Work-Energy Theorem, valid for particles with constant mass, is modified by the contribution coming from de variation of the intrinsic mass of the particle. Even when a conservative force acts on the particle with variable mass its total mechanical energy does not conserve while the intrinsic mass of the particle changes. In this case the variation of the kinetic energy depends on the path followed by the particle while its intrinsic mass varies in time.

We follow reference 9 where the authors derive the dynamic equation of the $\mathrm{CM}$ of a set of particles whose the total mass value, $M_{C M}(t)$, varies over time. In section 3 we discuss the dynamics of the case presented in this reference where the value of the mass of each particle belonging to the system is constant, but 
one of these particles leaves or enters the system making the value of the mass of its CM to change over time. To simplify the discussion of this topic in section 3 we consider the special case where initially we have two particles in a set $\mathcal{A}$ and that at end has only one particle. The results derived in section 3 are valid if the moving particle is leaving or entering into the set $\mathcal{A}$.

Equation (37) drives the dynamics of the CM with variable mass and on its r.h.s. we have a dependency on the velocity vector $\overrightarrow{\mathbf{v}}_{2}(t)$ of the moving particle in the set $\mathcal{A}$, but we show that this equation is invariant under the Galilean transformations in any inertial frame. We show that although the dynamic equation depends on the velocity vector of one particle, all inertial frames are equivalent under the Galilean transformations.

We also show in the case that the particles that belong to the set $\mathcal{A}$ are free particles, the linear momentum vector of the CM of this, $\overrightarrow{\mathbf{P}}_{C M}(t)$, does not conserve in any inertial frame during the interval of time when the value of its mass $M_{C M}(t)$ changes over time. To exemplify this non-conservation law of $\overrightarrow{\mathbf{P}}_{C M}(t)$ we consider the motion of two free particles with constant mass moving in a onedimensional trajectory.

The results presented in section 3 can be extended to the movement of a $\mathrm{CM}$ of a set with $N$ particles with constant mass, with $N \geq 2$, and one or more particles leaving or entering this system of particles. They also show that we must be more careful when applying conservation laws to the movement of extended particles with variable masses.

In Appendix $\mathrm{A}$ we present the solutions of the Newton's Second Law, in the inertial frame $\mathbf{S}$, for some types of force: $\overrightarrow{\mathbf{F}}_{R}(t)=\overrightarrow{\mathbf{0}}, \overrightarrow{\mathbf{F}}_{R}(t)=\overrightarrow{\mathbf{F}}$, with $\overrightarrow{\mathbf{F}}$ being a constant force, and $\overrightarrow{\mathbf{F}}_{R}(t)=\overrightarrow{\mathbf{P}}(t)$, where $\overrightarrow{\mathbf{P}}(t)$ is the weight force of the particle with variable intrinsic mass. We derive the expressions of the position, velocity and acceleration of this particle for arbitrary functions $m(t)$, and consider two special functions $m_{1}(t)$ and $m_{2}(t)$ to have some closed expressions of these vectors.

As a final comment, we must say that the results of the present article show that the dynamics of a variable mass body obtained from Newton's Second Law distinguishes two situations: $(i)$ a particle with intrinsically variable mass; (ii) a composite body made up of parts, whose masses are constant, that can come out or be added to it. The undergraduate student must know that it is not possible to mimic the dynamics of a particle with variable intrinsic mass by studying the temporal evolution of composite bodies whose mass varies over time.

\section{A. Newton's Second Law solutions for a particle with mass $m(t)$ under the action of special forces}

In this appendix we calculate the solutions of the Newton's Second Law 6a), in the inertial frame $\mathbf{S}$, for three simple physical situations. Equation 10 corresponds to rewriting eq. (6a) of the particle with mass $m(t)$ in terms of its acceleration vector $\overrightarrow{\mathbf{a}}$ in this frame $\mathbf{S}$,

$$
\overrightarrow{\mathbf{a}}(t)=-\left(\frac{d m(t)}{d t}\right) \frac{\overrightarrow{\mathbf{v}}(t)}{m(t)}+\frac{\overrightarrow{\mathbf{F}}_{R}(t)}{m(t)} .
$$

$\overrightarrow{\mathbf{v}}(t)$ is the velocity vector of this particle measured in the inertial frame $\mathbf{S} . \overrightarrow{\mathbf{F}}_{R}(t)$ is the net physical force that acts on this punctual body.

In this appendix we calculate the vectors: position $(\overrightarrow{\mathbf{r}}(t))$, velocity $(\overrightarrow{\mathbf{v}}(t))$ and acceleration $(\overrightarrow{\mathbf{a}}(t))$ of the particle with variable mass, in the inertial frame $\mathbf{S}$, for the following forces $\overrightarrow{\mathbf{F}}_{R}(t)$ :

(i) $\overrightarrow{\mathbf{F}}_{R}(t)=\overrightarrow{\mathbf{0}}$, for $t \in(-\infty,+\infty), \overrightarrow{\mathbf{0}}$ being the null vector:

In the inertial frame $\mathbf{S}$, the particle with mass $m(t)$ is in free motion since $\overrightarrow{\mathbf{F}}_{R}(t)=\overrightarrow{\mathbf{0}}$. In this case eq. 6 a gives the conservation of the linear momentum vector $\overrightarrow{\mathbf{p}}(t)$, $\overrightarrow{\mathbf{p}}(t)=m(t) \overrightarrow{\mathbf{v}}(t)$, in this inertial frame,

$$
\frac{d(m(t) \overrightarrow{\mathbf{v}}(t))}{d t}=\overrightarrow{\mathbf{0}} \Rightarrow \overrightarrow{\mathbf{v}}(t)=\frac{m\left(t_{0}\right)}{m(t)} \overrightarrow{\mathbf{v}}\left(t_{0}\right) .
$$

$t_{0}$ is a fixed time, that we choose, and $t \in(-\infty,+\infty)$. We assume that $m(t) \neq 0$ for the the entire time span.

Replacing the result (52) in the r.h.s. of eq. (51), with $\overrightarrow{\mathbf{F}}_{R}(t)=\overrightarrow{\mathbf{0}}$, we obtain the acceleration vector of the particle with mass $m(t)$,

$$
\overrightarrow{\mathbf{a}}(t)=-\frac{m\left(t_{0}\right)}{m^{2}(t)}\left(\frac{d m(t)}{d t}\right) \overrightarrow{\mathbf{v}}\left(t_{0}\right) .
$$

The acceleration $\overrightarrow{\mathbf{a}}(t)$ is parallel to the velocity $\overrightarrow{\mathbf{v}}(t)$ of the particle at any time while its mass is varying. $\overrightarrow{\mathbf{a}}(t)$ has the same (opposite) direction as $\overrightarrow{\mathbf{v}}(t)$ if the value of the mass is decreasing (increasing). The movement of the particle is one-dimensional for any dependence of the mass in the time $t, m(t)$.

To calculate the particle position vector with mass $m(t), \overrightarrow{\mathbf{r}}(t)$, we return to eq. 52 ,

$$
\begin{aligned}
\overrightarrow{\mathbf{v}}(t) & =\frac{d \overrightarrow{\mathbf{r}}(t)}{d t}=\frac{m\left(t_{0}\right)}{m(t)} \overrightarrow{\mathbf{v}}\left(t_{0}\right) \Rightarrow \\
\Rightarrow \overrightarrow{\mathbf{r}}(t) & =\overrightarrow{\mathbf{r}}\left(t_{0}\right)+m\left(t_{0}\right)\left(\int_{t_{0}}^{t} \frac{d t^{\prime}}{m\left(t^{\prime}\right)}\right) \overrightarrow{\mathbf{v}}\left(t_{0}\right),
\end{aligned}
$$

where $\overrightarrow{\mathbf{r}}\left(t_{0}\right)$ is the position vector of this particle at the fixed instant $t=t_{0}$, and $t_{0}$ being a moment that we choose in its motion. In particular we can take $t=0$, the initial time that we start following this particle.

Now we calculate the function $m(t)$ for which the acceleration $\overrightarrow{\mathbf{a}}(t)$ is a constant vector, see eq. (53), that is,

$$
-\frac{m\left(t_{0}\right)}{m^{2}(t)}\left(\frac{d m(t)}{d t}\right)=A,
$$

$A$ being a constant, $A \in \mathbb{R}$. The dimension of the constant $A$ is the inverse of the time: $[A]=T^{-1}$. 
The solution from eq. 55 is

$$
m_{A}\left(t ; t_{0}\right)=\frac{m\left(t_{0}\right)}{1+A\left(t-t_{0}\right)} .
$$

Now we consider two time dependencies of the mass of the particle, $m(t)$, to explicitly calculate the vectors $\overrightarrow{\mathbf{r}}(t), \overrightarrow{\mathbf{v}}(t)$ and $\overrightarrow{\mathbf{a}}(t)$ of the free particle with variable mass, $\overrightarrow{\mathbf{F}}_{R}(t)=\overrightarrow{\mathbf{0}}$, in the inertial frame $\mathbf{S}$. To simplify the expressions of these vectors, we take $t_{0}=0$.

$$
m_{1}(t) \equiv \begin{cases}m(0), & t<0 \\ m_{A}(t ; 0), & 0 \leq t_{1} \\ m_{A}\left(t_{1} ; 0\right), & t \geq t_{1}\end{cases}
$$

$A$ is a real constant. Due to the positivity of the mass $m(t)$ we have to have the condition: $1+A t_{1}>0$. The time $t_{1}$ only has to satisfy the condition to be greater than $0, t_{1}>0$. The $m_{1}(t)$ mass graph, with $A>0$, is shown in Fig. 5 a.

For $0 \leq t \leq t_{1}$ the mass varies over time and it is equal to the function $m_{A}(t ; 0)$ given by eq. (56). During this time interval Newton's Second Law is valid only in one inertial frame, the inertial frame $\mathbf{S}$. We do not have a recipe to determine which inertial frame is this particular inertial frame.

In the inertial frame $\mathbf{S}$, where Newton's Second Law 15 is valid, we have

$$
\frac{d\left(m_{1}(t) \overrightarrow{\mathbf{v}}(t)\right)}{d t}=\overrightarrow{\mathbf{0}},
$$

and from it it we obtain:

$$
\begin{aligned}
& \overrightarrow{\mathbf{a}}(t) \underbrace{=}_{(55)}=A \overrightarrow{\mathbf{v}}(0), \\
& \overrightarrow{\mathbf{v}}(t) \underbrace{==}_{(52)}=(1+A t) \overrightarrow{\mathbf{v}}(0), \\
& \overrightarrow{\mathbf{r}}(t) \underbrace{==}_{(54)}=\overrightarrow{\mathbf{r}}(0)+t \overrightarrow{\mathbf{v}}(0)+\frac{A t^{2}}{2} \overrightarrow{\mathbf{v}}(0) .
\end{aligned}
$$

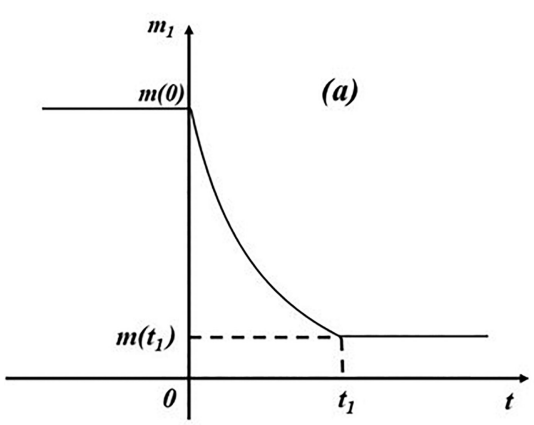

In this case, the free particle with variable mass $m_{1}(t), \overrightarrow{\mathbf{F}}_{R}(t)=\overrightarrow{\mathbf{0}}$, has a uniformly accelerated movement along the direction of the initial velocity $\overrightarrow{\mathbf{v}}(0)$ during the time interval that its mass changes.

( $i .2)$ Let us consider another time dependence for the variable intrinsics mass, $m_{2}(t)$,

$$
m_{2}(t) \equiv m_{0}(a+b \tanh (\alpha t)), \quad t \in(-\infty,+\infty) .
$$

The constant $m_{0}$ has a mass dimension, $\left[m_{0}\right]=M$, the constants $a$ and $b$ are dimensionless and the constant $\alpha$ has dimension of the inverse of the time, $[\alpha]=T^{-1}$. We assume that $a$ and $\alpha$ are positive real constants, and $b$ is also a real constant that can be negative or positive. To guarantee that the mass $m_{2}(t)$ is positive at any time, the constants $a$ and $b$ have to satisfy the condition:

$$
a-|b|>0 \text {. }
$$

In Fig. $5 \mathrm{p}$ you have the plot of $m_{2}(t)$ with $b>0$.

In the inertial frame $\mathbf{S}$, where Newton's Second Law (52) is valid, we substitute the expression of the mass $m_{2}(t)$ in the results $(53),(52)$ and $(54)$ to calculate the vectors $\overrightarrow{\mathbf{a}}(t), \overrightarrow{\mathbf{v}}(t)$ and $\overrightarrow{\mathbf{r}}(t)$, respectively, which describe the motion of this particle in the inertial frame $\mathbf{S}$,

$$
\begin{aligned}
\overrightarrow{\mathbf{a}}(t)= & -a b \alpha\left(\frac{\operatorname{sech}(\alpha t)}{a+b \tanh (\alpha t)}\right) \overrightarrow{\mathbf{v}}(0), \\
\overrightarrow{\mathbf{v}}(t)= & \left(\frac{a}{a+b \tanh (\alpha t)}\right) \overrightarrow{\mathbf{v}}(0), \\
\overrightarrow{\mathbf{r}}(t)= & \overrightarrow{\mathbf{r}}(0)+a\left[\frac{a t}{a^{2}-b^{2}}\right. \\
& \left.-\frac{b}{\alpha\left(a^{2}-b^{2}\right)} \ln \left(\frac{b \operatorname{senh}(\alpha t)+a \cosh (\alpha t)}{a}\right)\right] \overrightarrow{\mathbf{v}}(0) .
\end{aligned}
$$

The limits of $t \rightarrow \pm \infty$ of the vectors $\overrightarrow{\mathbf{a}}(t), \overrightarrow{\mathbf{v}}(t)$ and $\overrightarrow{\mathbf{r}}(t)$ describe a free particle with constant mass in a two different uniform one-dimensional movements.

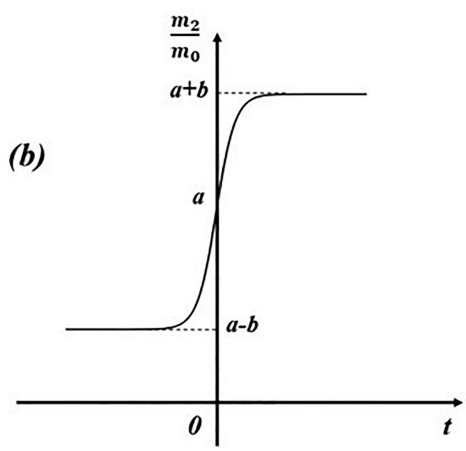

Figure 5: $(a)$ The mass shape graph $m_{1}(t)$ as a function of the time $t$, see eq. (57), with $A>0$ and $m(0)>0 ;(b)$ We draw the shape of the function $m_{2}(t)$ as a function of the time $t$, see eq. (59a), with $b>0$. The values of the constants $m_{0}, a$ and $\alpha$ are also positive. 
(ii) $\overrightarrow{\mathbf{F}}_{R}(t)=\overrightarrow{\mathbf{F}}, \overrightarrow{\mathbf{F}}$ being a constant force vector:

In this case, the law (15) that drives the motion of the particle with variable mass in the inertial frame $\mathbf{S}$ is

$$
\frac{d(m(t) \overrightarrow{\mathbf{v}}(t))}{d t}=\overrightarrow{\mathbf{F}}
$$

whose solution is

$$
\overrightarrow{\mathbf{v}}(t)=\frac{m\left(t_{0}\right)}{m(t)} \overrightarrow{\mathbf{v}}\left(t_{0}\right)+\left(\frac{t-t_{0}}{m(t)}\right) \overrightarrow{\mathbf{F}},
$$

where $t_{0}$ is a fixed time that we choose.

From the eq. 61a we obtain a relationship between the acceleration vector of the particle with mass $m(t)$ and its velocity,

$$
\overrightarrow{\mathbf{a}}(t)=-\frac{1}{m(t)}\left(\frac{d m(t)}{d t}\right) \overrightarrow{\mathbf{v}}(t)+\frac{\overrightarrow{\mathbf{F}}}{m(t)} .
$$

Replacing the result (61b) in the r.h.s. of the previous result, we obtain the expression of $\overrightarrow{\mathbf{a}}(t)$,

$$
\begin{aligned}
\overrightarrow{\mathbf{a}}(t)= & -\frac{m\left(t_{0}\right)}{m^{2}(t)}\left(\frac{d m(t)}{d t}\right) \overrightarrow{\mathbf{v}}\left(t_{0}\right) \\
& +\left[1-\frac{\left(t-t_{0}\right)}{m(t)}\left(\frac{d m(t)}{d t}\right)\right] \frac{\overrightarrow{\mathbf{F}}}{m(t)} .
\end{aligned}
$$

Since $\overrightarrow{\mathbf{v}}(t)=\frac{d \overrightarrow{\mathbf{r}}(t)}{d t}$, we calculate the position vector $\overrightarrow{\mathbf{r}}(t)$ of the result $61 \mathrm{~b}$,

$$
\begin{aligned}
\overrightarrow{\mathbf{r}}(t)=\overrightarrow{\mathbf{r}}\left(t_{0}\right)+ & +m\left(t_{0}\right) \overrightarrow{\mathbf{v}}\left(t_{0}\right)\left(\int_{t_{0}}^{t} \frac{d t^{\prime}}{m\left(t^{\prime}\right)}\right)+\frac{\left(t-t_{0}\right)^{2}}{2 m(t)} \overrightarrow{\mathbf{F}}+ \\
& +\left(\int_{t_{0}}^{t} \frac{\left(t^{\prime}-t_{0}\right)}{m^{2}\left(t^{\prime}\right)}\left(\frac{d m\left(t^{\prime}\right)}{d t^{\prime}}\right) d t^{\prime}\right) \frac{\overrightarrow{\mathbf{F}}}{2} \cdot(64)
\end{aligned}
$$

It is simple to verify that the expressions $61 \mathrm{~b}, 6$ and 64 for the particle with constant mass, $\frac{d m(t)}{d t}=0$, are reduced to the known vectors $\overrightarrow{\mathbf{v}}(t), \overrightarrow{\mathbf{a}}(t)$ and $\overrightarrow{\mathbf{r}}(t)$ when this body with constant mass is under the action of a constant force vector $\overrightarrow{\mathbf{F}}$.

From the result $(63)$ we notice that the acceleration vector of the particle with variable mass, $\overrightarrow{\mathbf{a}}(t)$, acquires a component in the direction of the velocity $\overrightarrow{\mathbf{v}}\left(t_{0}\right)$ while the value of the mass varies over time, in addition to the component of the acceleration towards the constant force $\overrightarrow{\mathbf{F}}$. The component of $\overrightarrow{\mathbf{a}}(t)$ in direction of $\overrightarrow{\mathbf{F}}$ also has a contribution from the variation of the inertia of the body.

As a final comment on the expression (64) of $\overrightarrow{\mathbf{r}}(t)$, we see that while its mass varies, the particle moves in the plane containing the vectors $\overrightarrow{\mathbf{v}}\left(t_{0}\right)$ and $\overrightarrow{\mathbf{F}}$ when they are not collinear vectors. When the vectors $\overrightarrow{\mathbf{v}}\left(t_{0}\right)$ and $\overrightarrow{\mathbf{F}}$ are collinear, the particle moves along a straight line during the time its mass varies.

(iii) $\overrightarrow{\mathbf{F}}_{R}(t)=m(t) \overrightarrow{\mathbf{g}}=\overrightarrow{\mathbf{P}}(t)$, the free fall of a particle with variable mass under the action of its weight:

We describe the motion of a particle of mass $m(t)$ that has an initial velocity vector $\overrightarrow{\mathbf{v}}(0)$ which is drawn

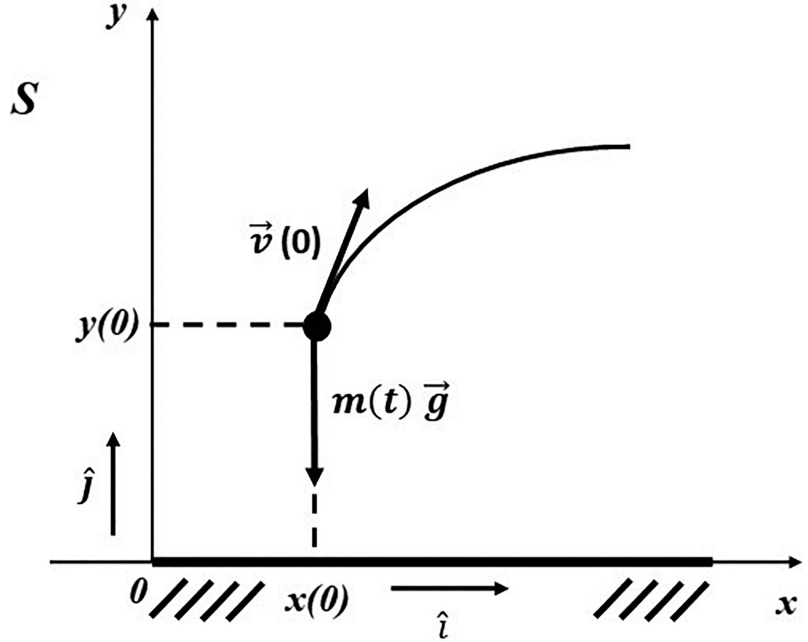

Figure 6: A particle with mass $m(t)$ is launched in a free falling motion from the initial position $\vec{r}(0)=x(0) \hat{\imath}+y(0) \hat{\jmath}$ and with velocity $\overrightarrow{\mathbf{v}}(0)$. The only force acting on the particle is its weight, $\overrightarrow{\mathbf{P}}(t)=m(t) \overrightarrow{\mathbf{g}} . \hat{\imath}(\hat{\jmath})$ is the unitary vector along the $x$-axis (y-axis).

in Fig. 6 The motion of this body is observed from the inertial frame $\mathbf{S}$, where Newton's Second Law (15) describes its movement,

$$
\begin{aligned}
\frac{d(m(t) \overrightarrow{\mathbf{v}}(t))}{d t} & =m(t) \overrightarrow{\mathbf{g}} \\
& =m(t) g \hat{\jmath} .
\end{aligned}
$$

We have $g<0$ and $\hat{\jmath}$ is the unit vector in the $y$ axis, see Fig. 6. The motion of the particle with mass $m(t)$ happens in the plane formed by the initial velocity vector $\overrightarrow{\mathbf{v}}(0)$ and the vertical direction, the $y$ axis.

Instead of completely solving the problem of a particle with a variable mass in free fall, with the general function $m(t)$, which has a long expression for the position vector and it does not give more insight on this problem, in the following we calculate the vector $\overrightarrow{\mathbf{v}}(t)$ and $\overrightarrow{\mathbf{a}}(t)$ in the inertial frame $\mathbf{S}$ for arbitrary $m(t)$ and the position vector $\overrightarrow{\mathbf{r}}(t)$ for the particular expression $m_{1}(t)$ for the particle's variable intrinsic mass.

We write the velocity vector at any time $t, \overrightarrow{\mathbf{v}}(t)$, in terms of its $x$ and $y$ components, see Fig. 6

$$
\overrightarrow{\mathbf{v}}(t)=v_{x}(t) \hat{\imath}+v_{y}(t) \hat{\jmath} .
$$

$\hat{\imath}(\hat{\jmath})$ is the unitary vector in the $x$-axis ( $y$-axis).

From eq. 65b), we have

$$
\begin{aligned}
\frac{d\left(m(t) v_{x}(t)\right)}{d t}= & 0 \Rightarrow v_{x}(t)=\frac{m(0)}{m(t)} v_{x}(0) \\
\frac{d\left(m(t) v_{y}(t)\right)}{d t}= & m(t) g \Rightarrow v_{y}(t)=\frac{m(0)}{m(t)} v_{y}(0) \\
& +\frac{g}{m(t)}\left(\int_{0}^{t} m\left(t^{\prime}\right) d t^{\prime}\right)
\end{aligned}
$$


When writing eqs. $67 \mathrm{a}$ and $67 \mathrm{~b}$ we choose $t_{0}=$ 0 to simplify the expressions of the vectors. We must remember that $g<0$.

Equation 65a relates the acceleration vector of the particle with mass $m(t), \overrightarrow{\mathbf{a}}(t)$, with its velocity, $\overrightarrow{\mathbf{v}}(t)$,

$$
\overrightarrow{\mathbf{a}}(t)=-\frac{1}{m(t)}\left(\frac{d m(t)}{d t}\right) \overrightarrow{\mathbf{v}}(t)+g \hat{\jmath} .
$$

Writing the acceleration $\overrightarrow{\mathbf{a}}(t)$ in terms of its $x$ and $y$ components, $a_{x}(t)$ and $a_{y}(t)$, respectively, that is

$$
\overrightarrow{\mathbf{a}}(t)=a_{x}(t) \hat{\imath}+a_{y}(t) \hat{\jmath},
$$

eq. 68) gives:

$$
\begin{aligned}
a_{x}(t)= & -\frac{m(0)}{m^{2}(t)}\left(\frac{d m(t)}{d t}\right) v_{x}(0) \\
a_{y}(t)= & -\frac{m(0)}{m^{2}(t)}\left(\frac{d m(t)}{d t}\right) v_{y}(0)+ \\
& +\left[1-\frac{1}{m^{2}(t)}\left(\frac{d m(t)}{d t}\right) \int_{0}^{t} m\left(t^{\prime}\right) d t^{\prime}\right] g .
\end{aligned}
$$

When we compare the motion of the particle in free fall when its mass is constant and when it (the mass) varies over time, we verify from the eqs. $67 \mathrm{a}$ and $70 \mathrm{a}$ that the $x$ component of the velocity vector $\overrightarrow{\mathbf{v}}(t)$ is not constant during the time when the intrinsic mass of the body varies. To have an horizontal component of the velocity in the movement of the particle in free fall, we must have $v_{x}(0) \neq 0$.

Equations $67 \mathrm{~b}$ and $70 \mathrm{~b}$ shows that the variation of the $y$ component of the velocity, $v_{y}(t)$, depends on the weight force of the particle, $\overrightarrow{\mathbf{P}}(t)=m(t) \overrightarrow{\mathbf{g}}$, but it also depends on the initial value of $v_{y}(t)$, that is, $v_{y}(0) \neq 0$. We do not have this last dependence on $v_{y}(t)$ in the expression of $a_{y}(t)$ if the mass of the particle is constant.

The $g$-term in the component $a_{y}(t)$, see eq. $70 \mathrm{~b}$, has a contribution coming from the variation of the value of the mass $m(t)$ over time.

To end this appendix, we assume that $m(t)=$ $m_{A}(t ; 0)$, see the expression $(56)$. In this case we obtain,

$$
\begin{aligned}
& a_{x}(t)=A v_{x}(0) \\
& a_{y}(t)=g+A v_{y}(0)+g \ln (1+A t), \\
& v_{x}(t)=(1+A t) v_{x}(0), \\
& v_{y}(t)=(1+A t) v_{y}(0)+\frac{g}{A}(1+A t) \ln (1+A t) .
\end{aligned}
$$

The position vector of the particle with mass $m_{A}(t ; 0)$, $\overrightarrow{\mathbf{r}}(t)$, has components $x$ and $y$, that is,

$$
\overrightarrow{\mathbf{r}}(t)=x(t) \hat{\imath}+y(t) \hat{\jmath} .
$$

From the results $\sqrt{71 c}$ and $(71 d)$ we calculate the two components of $\overrightarrow{\mathbf{r}}(t)$ of a particle of mass $m_{A}(t ; 0)$ in free fall,

$$
\begin{aligned}
v_{x}(t)= & \frac{d x(t)}{d t} \Rightarrow x(t)=x(0)+v_{x}(0) t+\frac{A t^{2}}{2} v_{x}(0), \\
v_{y}(t)= & \frac{d y(t)}{d t} \Rightarrow y(t)=y(0)+\left(t+\frac{A t^{2}}{2}\right) v_{y}(0)+ \\
& +\frac{g}{2 A^{2}}\left[\frac{1}{2}-\frac{(1+A t)^{2}}{2}+(1+A t)^{2} \ln (1+A t)\right] .
\end{aligned}
$$

The mass $m_{A}(t ; 0)$, see the expression 56 of this mass, has to satisfy the condition:

$$
1+A t>0
$$

where $A \in \mathbb{R}$. Note that the results $(71 \mathrm{a}-(71 \mathrm{~d})$, and (73a) and $73 \mathrm{~b}$ are independent of the initial value $m(0)$ of the mass $m_{A}(t ; 0)$.

In the limit of $A \rightarrow 0, x(t)$ and $y(t)$ recover the expressions of the movement of a particle, with constant mass, in free fall,

$$
\begin{aligned}
& \lim _{A \rightarrow 0} x(t)=x(0)+v_{x}(0) t, \\
& \lim _{A \rightarrow 0} y(t)=y(0)+v_{y}(0) t+\frac{t^{2}}{2} g .
\end{aligned}
$$

\section{References}

[1] I. Newton, Philosophiae Naturalis Principia Mathematica (Jussu Societatis Regiae ac typis Josephi Streater, London, 1687).

[2] A. Sommerfeld, Mechanics, Lectures on Theoretical Physics (Academic Press, New York, 1952), v. 1.

[3] J.F. Thorpe, Am. J. of Phys. 30, 637 (1962).

[4] M.S. Tiersten, Am. J. of Phys. 37, 82 (1969).

[5] J. Matolyak and G. Matous, Phys. Teach. 28, 328 (1990).

[6] C.A. de Souza and V.H. Rodrigues, Eur. J. Phys. 25, 41 (2004).

[7] K. Nakayama, Eur. J. Phys. 39, 055002 (2018).

[8] A.R. Plastino and J.C. Muzzio, Celest. Mech. Dyn. Astron. 53, 227 (1992).

[9] M.T. Thomaz and E.V. Corrêa Silva, Rev. Bras. Ensino de Física 41, e20180344 (2019).

[10] A.P. French, Newtonian Mechanics (The M.I.T. Introductory Physics Series) (W.W. Norton \& Company Inc, New York, 1971).

[11] S.T. Thornton and J.B. Marion, Classical Dynamics of Particles and Systems (Thomson Brooks, Belmont, 2003), $5^{\text {th }}$ ed.

[12] A.B. Arons and A.M. Bork, Am. J. of Phys. 32646 (1964).

[13] H.M. Nussenzveig, Curso de Física, 1: Mecânica (Blucher, São Paulo, 2013), $5^{\mathrm{a}}$ ed. 